\title{
Transcriptomic Study of Early Responses to the Bud Dormancy-breaking Agent Hydrogen Cyanamide in 'TropicBeauty' Peach
}

\author{
Lisa Tang, Shweta Chhajed, and Tripti Vashisth ${ }^{1}$ \\ Citrus Research and Education Center, University of Florida/IFAS, 700 Experiment Station Road \\ Lake Alfred, FL 33850 \\ Mercy A. Olmstead and James W. Olmstead \\ Horticultural Sciences Department, University of Florida, P.O. Box 110690, Gainesville, FL 32611
}

Thomas A. Colquhoun

Environmental Horticulture Department, University of Florida, P.O. Box 110670, Gainesville, FL 32611

\begin{abstract}
AdDitional INDEX wORDs. budbreak, endodormancy, low-chill cultivar, oxidative stress, RNA sequencing
ABstract. To determine how the dormancy-breaking agent hydrogen cyanamide (HC) advances budbreak in peach (Prunus persica), this study compared the transcriptome of buds of low-chill 'TropicBeauty' peach trees treated with $1 \%(\mathrm{v} / \mathrm{v}) \mathrm{HC}$ and that of nontreated trees at 3 and 7 days after treatment (DAT), respectively, using an RNA sequencing analysis. The peak of total budbreak occurred 6 weeks earlier in the HC-treated trees (at 32 DAT) than the nontreated trees (at 74 DAT). There were 1312 and 1095 differentially expressed genes (DEGs) at 3 and 7 DAT, respectively. At 3 DAT, DEGs related to oxidative stress, including the response to hypoxia, lipid oxidation, and reactive oxygen species (ROS) metabolic process, were upregulated in HC-treated buds. Additionally, DEGs encoding enzymes for ROS scavenging and the pentose phosphate pathway were upregulated at 3 DAT but they were not differently expressed at 7 DAT, indicating a temporary demand for defense mechanisms against HC-triggered oxidative stress. Upregulation of DEGs for cell division and development at 7 DAT, which were downregulated at 3 DAT, suggests that cell activity was initially suppressed but was enhanced within 7 DAT. At 7 DAT, DEGs related to cell wall degradation and modification were upregulated, which was possibly responsible for the burst of buds. The results of this study strongly suggest that $\mathrm{HC}$ induces transient oxidative stress shortly after application, leading to the release of bud dormancy and, subsequently, causing an increase in cell activity and cell wall loosening, thereby accelerating budbreak in peach.
\end{abstract}

During the fall in temperate climates, buds of deciduous woody perennials, including peach, enter dormancy, which is a state in which the visible growth of a plant structure containing a meristem is absent (Lang et al., 1987). Bud dormancy has two steps, endodormancy and ecodormancy, which are caused by internal tree factors and controlled by environmental conditions, respectively (Considine and Considine, 2016; Lang et al., 1987). When buds are in endodormancy, or "true dormancy," the meristem remains resting, even if the environmental conditions are optimal for growth resumption (Considine and Considine, 2016; Rohde and Bhalerao, 2007). The release of endodormancy requires cumulative exposure to significant low temperatures during winter; this required duration of low temperatures for dormancy-breaking is termed the chilling requirement and is specific to plant species and cultivars (Considine and Considine, 2016). Dormancy release occurs only when the chilling requirement is fulfilled; as a result, vegetative and flower buds start to swell and burst ("budbreak"), followed by vegetative growth or flowering and subsequent fruit development (Beauvieux et al., 2018; Dennis, 2003). Therefore, the timing of dormancy release and budbreak is critical for the onset of fruit production and the market window.

Received for publication 20 Mar. 2019. Accepted for publication 1 May 2019. ${ }^{1}$ Corresponding author. E-mail: tvashisth@ufl.edu.
For peach production in subtropic climates such as the southeastern United States, low-chill cultivars, which require less chill accumulation than those originating from temperate climates, have been developed (Okie, 1998; Olmstead et al., 2016). Nevertheless, the mild winter temperatures in subtropical areas are often insufficient to fulfill the chilling requirement of low-chill cultivars, thereby resulting in nonuniform budbreak and poor fruit sets (George et al., 1992; Olmstead, 2014). The use of plant growth regulators containing the active ingredient of hydrogen cyanamide (HC) to break bud dormancy and to achieve uniform budbreak has been documented for peach (George et al., 1992; Olmstead, 2014; Yamane et al., 2011) and a number of deciduous fruit crops, such as apple (Malus domestica), grapevine (Vitis vinifera), kiwifruit (Actinidia deliciosa), sweet cherry (Prunus avium), and both highbush blueberry (Vaccinium corymbosum hybrid) and rabbiteye blueberry ( $V$. ashei) (Bound and Jones, 2004; Godini et al., 2008; Henzell et al., 1992; Ionescu et al., 2017b; Jackson and Bepete, 1995; Shulman et al., 1986; Williamson et al., 2002).

Although the use of $\mathrm{HC}$ is well-documented, the underlying mechanism of its effects on budbreak is not completely understood. For grapevine, a reduction in catalase transcript levels and activity and the accumulation of the reactive oxygen species (ROS), including hydrogen peroxide, superoxide, and nitric oxide, were observed in buds soon after exogenous HC 
application, with a higher budbreak rate compared with that of the nontreated control (Halaly et al., 2008; Nir et al., 1986; Or et al., 2002; Sudawan et al., 2016). Furthermore, HC resulted in a rapid decrease in the consumption of oxygen in mitochondria and the temporary induction of alcohol dehydrogenase (ADH) and pyruvate decarboxylase (PDH) expressions in buds of grapevine, indicating the disturbance of aerobic respiration by HC (Halaly et al., 2008; Or et al., 2000; Pérez et al., 2009). The application of $\mathrm{HC}$ also reduced the expression of dormancyassociated MADS-box (DAM) genes DAM5 and DAM6, which are two of six $D A M$ genes isolated in the nondormant peach mutant with a role in dormancy maintenance (Bielenberg et al., 2008; Jiménez et al., 2010; Rodriguez-A et al., 1994), in peach buds at 2 and 4 weeks after treatment (Yamane et al., 2011). These results led to a generally accepted hypothesis that exogenous HC application results in transient oxidative stress that, at least in part, induces the release of bud dormancy and budbreak in deciduous crops (Beauvieux et al., 2018; Halaly et al., 2008; Or et al., 2000). However, this proposed mode of action has not yet been studied in peach.

In recent years, the peach industry in subtropical Florida has grown substantially with the development of low-chill cultivars as an alternative crop to Citrus species (Morgan and Olmstead 2013; Singerman et al., 2017), but the effects of exogenously applied HC have never been investigated in low-chill peach cultivars at transcript levels. Better knowledge of the mechanism of HC breaking bud dormancy is fundamental to develop strategies to efficiently manipulate the time of budbreak using plant growth regulators, thereby promoting uniform flowering and fruiting for commercial peach production. Therefore, the goal of this research was to determine the effect of $\mathrm{HC}$ treatment at the transcriptome level and the underlying mechanism that leads to early budbreak in peach. The responses of exogenous $\mathrm{HC}$ application were assessed by comparing the transcriptome using RNA sequencing in buds of trees treated with $1 \%(\mathrm{v} / \mathrm{v}) \mathrm{HC}$ and those of nontreated trees of peach cultivar TropicBeauty, which has a chilling requirement of 150 chill hours. The results provide evidence that HC resulted in oxidative stress in buds shortly after treatment, followed by the induction of cell growth and development and advanced budbreak for the low-chill peach cultivar grown in the subtropical climate.

\section{Materials and Methods}

Plant materials and HC treatment. Five-year-old 'TropicBeauty' peach, which have a chilling requirement of 150 chill hours, on 'Flordaguard' rootstock in an experimental orchard (Citra, FL) were used for this research. Chill hours for the duration of the experiment were calculated based on the Weinberger model (Weinberger, 1950), starting from 1 Oct. 2015 ( $\approx 2$ months before the experiment). The experiment had a complete randomized design, with two treatments replicated on four individual trees per treatment $(n=4)$. In Florida, commercial peach growers usually apply $\mathrm{HC}$ around midDecember to promote uniform and early fruit sets. Therefore, on 16 Dec. 2015 (11 chill hours), one set of trees was sprayed with 1\% (v/v) HC (BudPro; Green Trees \& Plants II, Woodstock, GA) containing $0.15 \%$ (v/v) nonionic surfactant (Silwet L-77; Helena Agri-Enterprises, Collierville, TN) with a backpack sprayer until dripping; the other set of trees was not treated with HC (they were sprayed with water with surfactant) and was referred to as the control trees during this research.
Ten buds of each of the four control and HC-treated trees (as four biological replicates) were collected at 0 (pretreatment), 1 , 3 , and 7 DAT, respectively. Upon collection, bud samples were immediately frozen in liquid nitrogen and stored at $-80{ }^{\circ} \mathrm{C}$ until analysis.

Budbreak evaluation. Starting on 7 Jan. 2016 (21 DAT), the number of vegetative and flower buds that were swollen (beginning to open) or open on the four branches of uniform size per tree was quantified every 4 to $7 \mathrm{~d}$ until 15 Feb. 2016 (60 DAT) for HC-treated trees and until 7 Mar. 2016 (81 DAT) for control trees. The budbreak rate was expressed as the average number of swollen or open buds per branch per tree for the individual trees (replications) per treatment.

RNA EXTRACTION AND RNA SEQUENCING ANALySis. Total RNA was extracted using an RNA isolation kit (Plant/Fungi Total RNA Purification Kit; Norgen Biotek, Thorold, ON, Canada). The quality and quantity of RNA were evaluated using a spectrophotometer (Epoch 2 Microplate; BioTek Instruments, Winooski, VT) and denaturing formaldehyde 1.2\% agarose gels (Rio, 2015).

To determine the early response to $\mathrm{HC}$ in peach buds at a transcriptomic level, RNA samples of control and HC-treated trees at 3 and 7 DAT $(n=4)$ were sent to the Interdisciplinary Center for Biotechnology Research at the University of Florida (Gainesville, FL) for RNA sequencing (RNA-seq), alignment, and differential expression analysis. The Illumina TruSeq mRNA protocol (Illumina, San Diego, CA) was performed for transcript sequencing. The sequenced transcripts were annotated using the Prunus persica v2.1 genome from the Phytozome database (Verde et al., 2017). The RNA-seq read data are summarized in Supplemental Table 1. Differential gene expression was analyzed using RSEM v1.2.31 ( $\mathrm{Li}$ and Dewey, 2011). An absolute value of the $\log _{2}$ fold change greater than 1 and a false discovery rate (FDR)-corrected $P<$ 0.05 were used as the thresholds to identify the significantly differentially expressed genes (DEGs) of trees (HC-treated vs. control trees) at 3 and 7 DAT, respectively. The data discussed in this publication were deposited in the National Center for Biotechnology Information's Gene Expression Omnibus (GEO) (Barrett et al., 2013; Edgar et al., 2002) and are accessible through GEO Series accession number GSE128158.

GENE ONTOLOGY ENRICHMENT ANALYSIS OF DIFFERENTIALLY EXPRESSED GENES. To investigate the biological significance of the results of RNA-seq, DEGs at 3 and 7 DAT, respectively, were subjected to functional analyses. MapMan software (version 3.5.1.R2) (Thimm et al., 2004; Usadel et al., 2005) was used to identify the physiological or biochemical processes represented by the DEGs; only the statistically significant functional categories (BINs) based on the Wilcoxon rank sum test $(P<0.05)$ are reported here. A gene ontology (GO) enrichment analysis was performed using AgriGO, which uses Fisher's exact test (Tian et al., 2017). To obtain the results of the GO analysis, the GO terms and FDR according to multi-test adjustments more than 0.05 were discarded, leaving only statistically significant GO terms $(P<0.05)$. Redundant GO terms were further removed using REViGO (Supek et al., 2011). The enrichment values and the absolute value of the $\log _{10}$ FDR-adjusted $P$ value (Huang et al., 2009) of each significant GO term for the biological process, molecular function, and cellular component were plotted on heatmaps in which upregulated and downregulated DEGs were expressed as positive and negative, respectively, using Morpheus software 
(Broad Institute, Cambridge, MA). Additionally, the Kyoto Encyclopedia of Genes and Genomes (KEGG) Mapper tool (Kanehisa et al., 2017) was used to determine the metabolic pathways that involve the enzymes encoded by DEGs.

ANALYSIS OF GENE EXPRESSION USING REAL-TIME QUANTITATIVE POLYMERASE CHAIN REACTION. To validate the results of RNA-seq analysis, the relative expressions of formate dehydrogenase [FDH (Prupe.3G284000)], manganese superoxide dismutase1 [MSD1 (Prupe.2G262400)], glutathione s-transferase tau8 [GSTU8 (Prupe.6G264900)], glutathione $s$ transferase tau19 [GSTU19 (Prupe.4G147400)], peptide methionine sulfoxide reductase1 [PMSR1 (Prupe.5G161000)], and nadph:quinone oxidoreductase [NQR (Prupe.2G038400)] selected from the DEGs were determined using real-time quantitative polymerase chain reaction (qPCR). In addition, $D A M 1$, $D A M 5, D A M 6$, apetalal (AP1), pistillata $(P I)$, agamous $(A G)$, and floral binding protein 9 (FBP9) were analyzed because their expressions were strongly related to dormancy or flowering in peach (Bielenberg et al., 2008; Jiménez et al., 2010; Tani et al., 2009; Zhang et al., 2008). Gene-specific primers were designed using Primer BLAST (National Center for Biotechnology Information, 2018). The sequences of the primers are listed in Supplemental Table 2.

For cDNA synthesis, $1 \mu \mathrm{g}$ of total RNA of the buds collected at $0,1,3$, and 7 DAT, respectively, was first treated with DNase I (RQ1; Promega, Madison, WI) and used for first-strand synthesis using oligo $(\mathrm{dT})_{15}$ primer, dNTP mix, and reverse transcriptase (ImProm-II; Promega) in a $20-\mu \mathrm{L}$ reaction according to the manufacturer's protocol.

A real-time PCR system (7500 Fast Real-Time PCR System; Applied Biosystems, Foster City, CA) was used to perform qPCR in a $10-\mu \mathrm{L}$ reaction system containing $10 \mathrm{ng}$ of cDNA (calculated from RNA), $300 \mathrm{nM}$ forward and reverse primers, and SYBR green reagent mix [PowerUp SYBR Green Master Mix (2X); Applied Biosystems]. Each reaction was performed at $50{ }^{\circ} \mathrm{C}$ for $2 \mathrm{~min}$ and $95^{\circ} \mathrm{C}$ for $2 \mathrm{~min}$, followed by 40 cycles of $95{ }^{\circ} \mathrm{C}$ for $3 \mathrm{~s}$ and $60{ }^{\circ} \mathrm{C}$ for $30 \mathrm{~s}$. Dissociation curve analysis ranging from 60 to $95{ }^{\circ} \mathrm{C}$ was performed at the end of each qPCR to confirm that nonspecific products were not formed.

Using the quantification cycle $(\mathrm{Ct})$, the levels of relative expression (fold change) of genes of interest were calculated using the Pfaffl method (Hellemans et al., 2007; Pfaffl, 2001) with translation elongation factor 2 (TEF2) and ubiquitin $(U B Q)$ as the reference genes (Tong et al., 2009; Yamane et al., 2011). The two reference genes were selected among other housekeeping genes tested preliminarily (translation elongation factor 1-alpha, 18 s ribosomal RNA, RNA polymerase II, $\alpha$-tubulin, $\beta$-tubulin, and actin 2/7) based on their stability across bud samples collected on different days. Gene expression data for each sampling date were the mean of four biological replicates; each biological replicate was the mean of two qPCR (technical) replicates.

Statistical analysis. Student's $t$ test was used to test for the treatment effect of $\mathrm{HC}$ on the budbreak rate and relative gene expression levels (qPCR results) using SAS (version 9.3; SAS Institute, Cary, NC). An analysis of variance (ANOVA) was used to determine the changes in the budbreak rate over time using the General Linear Model procedure of SAS; when ANOVA testing indicated significant differences, post hoc comparisons were performed using Tukey's honestly significant difference (HSD) procedure with a family error rate of $\alpha \leq$ 0.05. Pearson's correlation coefficients were calculated to identify significant relationships $(r>0.5 ; P \leq 0.05)$ between relative gene expression quantified with qPCR and fold change from the RNA-seq analysis.

\section{Results}

EFFECTS OF HC ON BUDBREAK. For nontreated control trees, only a few buds ( $<3$ buds/branch) had emerged within 60 DAT (Fig. 1). The maximum budbreak rate over time occurred at 74 and 81 DAT for the control trees $(P<0.001)$. In contrast, a substantial number of swollen or open buds ( $\approx 10$ buds/branch) were observed in HC-treated trees starting at 21 DAT and the budbreak rate reached its maximum at 32 and 39 DAT $(P<$ $0.001)$, which was 6 weeks earlier than that of the nontreated trees. For HC-treated trees, the reduction in the budbreak rate after 39 DAT was due to the reduction in flower buds as a result of fruit setting following bloom. There was no significant difference in the number of vegetative buds, flower buds, or total buds on the day when the maximum budbreak occurred (39 DAT for HC-treated trees and 81 DAT for control) for HCtreated and control trees, indicating that $\mathrm{HC}$ treatment resulted in early budbreak without suppressing the budbreak rate.

Analysis of Differentially EXPRESSION Genes. Differences in the transcriptome of the buds of HC-treated trees compared with the nontreated control at 3 and 7 DAT were obtained using RNA-seq analysis. There were 1312 and 1095 DEGs at 3 and 7 DAT, respectively, with absolute values of fold change greater than 1 and FDR less than 0.05. For DEGs at 3 DAT, 610 were upregulated and 702 were downregulated in buds of HC-treated trees compared with nontreated trees, whereas there were 689 and 406 upregulated and downregulated, respectively, DEGs at 7 DAT of HC-treated trees. Only 88 DEGs were common between the two sampling days; among them, 40 were upregulated and 48 were downregulated in buds of treated trees in comparison with those of nontreated trees. The extracellular region (GO:0005576) was the only significant GO term for the 40 common upregulated DEGs. For the DEGs that were commonly downregulated at both 3 and 7 DAT, significant GO terms were related to stress responses, including the response to abiotic stimulus (GO:0009628), response to stimulus (GO:0050896), response to chemical stimulus (GO:0042221), secondary metabolic process (GO:0019748), and response to temperature stimulus (GO:0009266).

EnRichment anAlysis of MAPMAN fUnCtional CATEgories. The enrichment analysis of gene categories using MapMan software provided an overview of the physiological and biochemical processes of DEGs at 3 and 7 DAT, respectively. The results of the MapMan analysis demonstrated that most or all DEGs in the metabolic pathways involving nitrile lyases and nitrilases [BIN $26.8(P=0.010)]$ and glutathione S-transferases (GST) [BIN $26.9(P=0.005)]$ were upregulated at 3 DAT (Table 1). The majority of DEGs for regulating DNA [BIN 28 $(P<0.001)$ ], DNA synthesis and chromatin structure [BIN 28.1 $(P<0.001)]$, and kinase-involved post-translational modifications [BIN29.4.1 $(P=0.048)]$ were downregulated at 3 DAT. Interestingly, DEGs involved in the photosynthesis pathway [BIN $1(P=0.018)]$ were upregulated at 3 DAT; nevertheless, this result was unexpected because no visible green photosynthetic tissues had emerged from the bud scales at 3 DAT for HC-treated trees. At 7 DAT, most DEGs involving the regulation of DNA [BIN $28(P=0.002)]$, DNA synthesis and 
chromatin structure [BIN $28.1(P=0.002)]$, kinase-involved post-translational modifications [BIN29.4.1 $(P=0.019)]$, and cell organization [BIN $31.1(P=0.029)$ ] were upregulated, as opposed to the results at 3 DAT. There were several upregulated DEGs assigned to cell wall degradation [BIN 10.6 $(P=$ 0.031)].

ENRICHMENT ANALYSIS OF GENE ONTOLOGY. To obtain an interpretation of the more recent assignment of gene function, a GO enrichment analysis was performed with upregulated and downregulated DEGs, respectively, at 3 and 7 DAT. The significant GO terms analyzed using AgriGO and REViGO were visualized as heatmaps for biological processes, molecular functions, and cellular components (Supplemental Fig. 1). Regarding biological processes, 238 significant GO terms were

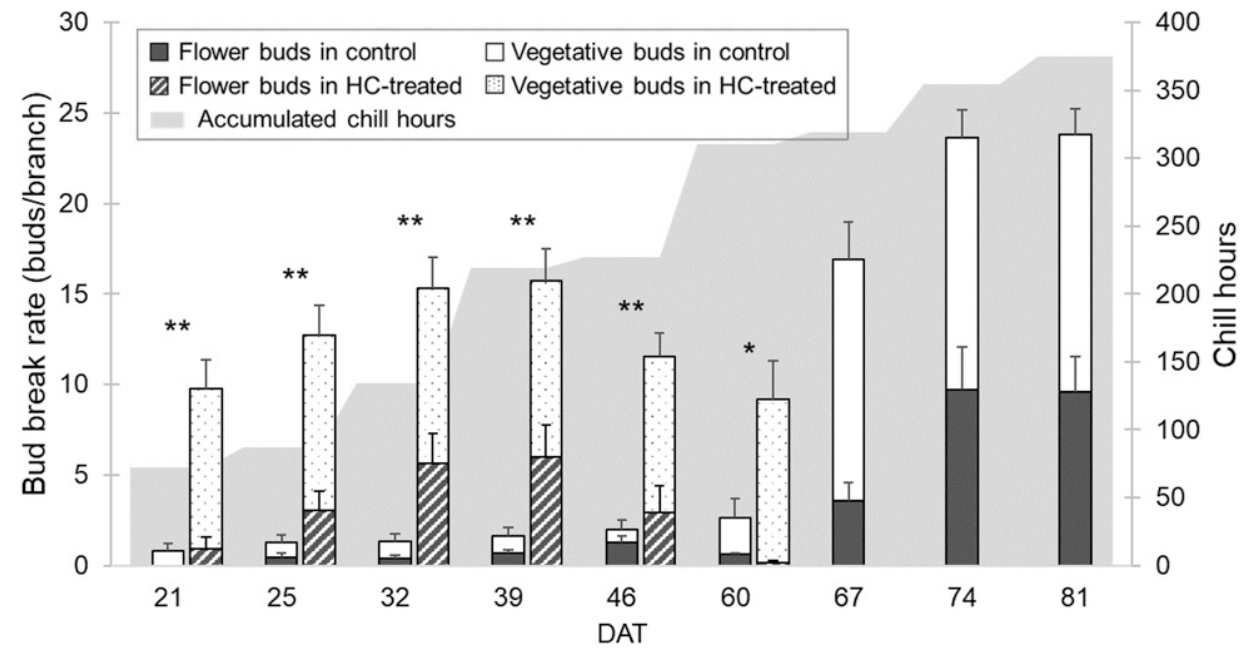

Fig. 1. Budbreak rate expressed as the number of swollen (about to open) or open flower and vegetative buds for nontreated 'TropicBeauty' peach control trees and 'TropicBeauty' peach trees treated with $1 \%(\mathrm{v} / \mathrm{v})$ hydrogen cyanimide (HC) 21 to $81 \mathrm{~d}$ after treatment (DAT) and 21 to 60 DAT, respectively, and the accumulation of chill hours (Weinberger, 1950) in Citra, FL, since 1 Oct. 2015. Data are means \pm SE of four biological replicates. *Significant differences based on Student's $t$ test for total budbreak rate between control and HC-treated trees on the same day. further categorized into two major groups (groups 1 and 2) based on the patterns of DEGs on the two sampling days (Table 2). Group 1 included the two subgroups of GO terms in which DEGs had decreasing expression patterns from 3 to 7 DAT. For the GO terms of subgroup 1A, DEGs were upregulated at 3 DAT; however, these GO terms were not statistically significant at 7 DAT. The GO terms of subgroup 1B were not significant at 3 DAT; however, DEGs were downregulated at 7 DAT. In contrast to group 1, the expression of DEGs in the GO terms assigned to group 2 increased over time. Group 2 was further divided into three subgroups of GO terms: GO terms that consisted of downregulated and upregulated DEGs at 3 and 7 DAT, respectively (subgroup 2A); GO terms with downregulated DEGs at 3 DAT but that were not significant at 7 DAT (subgroup 2B); and GO terms that were nonsignificant at 3 DAT but contained upregulated DEGs at 7 DAT.

The GO terms for which DEGs had decreasing patterns (group 1) included response to oxidative stress (GO:0006979), response to hypoxia (GO:0001666), respiratory burst (GO:0045730), oxygen and ROS metabolic process (GO:0006800), hydrogen peroxide metabolic process (GO:0042743), and lipid oxidation (GO:0034440) (Table 2). In addition, a number of DEGs related to antioxidant activity were upregulated at 3 DAT (Table 3 ), suggesting the activation of defense mechanisms against oxidative stress. Death (GO:0016265) and cell death (GO:0008219) were also included in group 1. Consistently, group 2 included the GO terms related to growth and development,

Table 1. Selected significant categories (BINs) of MapMan enrichment analysis (Thimm et al., 2004; Usadel et al., 2005) for differentially expressed genes (DEGs) in buds of 'TropicBeauty' peach trees treated with $1 \%(\mathrm{v} / \mathrm{v})$ hydrogen cyanamide compared with nontreated control trees at 3 and $7 \mathrm{~d}$ after treatment (DAT).

\begin{tabular}{|c|c|c|c|c|c|}
\hline DAT & $\mathrm{BIN}$ & Function & Upregulated DEGs (no.) & Downregulated DEGs (no.) & $P$ \\
\hline 3 & 1 & Photosynthesis & 20 & 2 & 0.018 \\
\hline 3 & 26.8 & $\begin{array}{l}\text { Misc. nitrilases, nitrile lyases, berberine bridge enzymes, } \\
\text { reticuline oxidases, troponine reductases }\end{array}$ & 6 & 1 & 0.010 \\
\hline 3 & 26.9 & Misc. glutathione s-transferases & 6 & 0 & 0.005 \\
\hline 3 & 26.24 & Misc. GCN5-related n-acetyltransferase & 4 & 0 & 0.037 \\
\hline 3 & 26.28 & Misc. GDSL-motif lipase & 1 & 6 & 0.042 \\
\hline 3 & 28 & DNA & 5 & 23 & 0.001 \\
\hline 3 & 28.1 & DNA synthesis/chromatin structure & 1 & 21 & $<0.001$ \\
\hline 3 & 29.4 .1 & Protein post-translational modification kinase & 0 & 3 & 0.048 \\
\hline 7 & 10 & Cell wall & 25 & 5 & 0.050 \\
\hline 7 & 10.6 & Cell wall degradation & 10 & 2 & 0.031 \\
\hline
\end{tabular}


Table 2. Selected significant gene ontology (GO) terms of differentially expressed genes (DEGs) with enrichment values (EV) in buds of 'TropicBeauty' peach trees treated with $1 \%(\mathrm{v} / \mathrm{v})$ hydrogen cyanamide compared with nontreated control trees at 3 and $7 \mathrm{~d}$ after treatment (DAT) in the aspect of GO biological process.

\begin{tabular}{|c|c|c|c|c|}
\hline \multirow[b]{2}{*}{ GO term } & \multicolumn{2}{|c|}{3 DAT } & \multicolumn{2}{|c|}{7 DAT } \\
\hline & EV & DEGs (no.) & $\overline{\mathrm{EV}}$ & DEGs (n \\
\hline \multicolumn{5}{|l|}{ Group 1} \\
\hline \multicolumn{5}{|l|}{ Subgroup 1A } \\
\hline GO:0006952 defense response & $10.7^{z}$ & 76 & 0.0 & 0 \\
\hline GO:0055114 oxidation reduction & 10.7 & 68 & 0.0 & 0 \\
\hline $\begin{array}{l}\text { GO:0006800 oxygen and reactive oxygen species } \\
\text { metabolic process }\end{array}$ & 5.4 & 24 & 0.0 & 0 \\
\hline GO:0042743 hydrogen peroxide metabolic process & 5.1 & 23 & 0.0 & 0 \\
\hline GO:0034641 cellular nitrogen compound metabolic process & 5.0 & 53 & 0.0 & 0 \\
\hline GO:0010310 regulation of hydrogen peroxide metabolic process & 4.4 & 16 & 0.0 & 0 \\
\hline $\begin{array}{l}\text { GO:0080010 regulation of oxygen and reactive oxygen species } \\
\text { metabolic process }\end{array}$ & 4.3 & 16 & 0.0 & 0 \\
\hline GO:0045730 respiratory burst & 4.3 & 13 & 0.0 & 0 \\
\hline GO:0044255 cellular lipid metabolic process & 4.2 & 51 & 0.0 & 0 \\
\hline GO:0008219 cell death & 3.6 & 25 & 0.0 & 0 \\
\hline GO:0009626 plant-type hypersensitive response & 3.6 & 22 & 0.0 & 0 \\
\hline GO:0016265 death & 3.6 & 25 & 0.0 & 0 \\
\hline GO:0012501 programmed cell death & 3.3 & 23 & 0.0 & 0 \\
\hline GO:0070482 response to oxygen levels & 2.4 & 9 & 0.0 & 0 \\
\hline GO:0048583 regulation of response to stimulus & 2.3 & 26 & 0.0 & 0 \\
\hline GO:0006979 response to oxidative stress & 2.1 & 23 & -2.3 & 20 \\
\hline GO:0034440 lipid oxidation & 1.4 & 10 & 0.0 & 0 \\
\hline GO:0001666 response to hypoxia & 1.3 & 7 & 0.0 & 0 \\
\hline \multicolumn{5}{|l|}{ Subgroup 1B } \\
\hline GO:0009266 response to temperature stimulus & 0.0 & 0 & -3.0 & 30 \\
\hline GO:0009642 response to light intensity & 0.0 & 0 & -2.6 & 14 \\
\hline GO:0009644 response to high light intensity & 0.0 & 0 & -2.3 & 12 \\
\hline GO:0031326 regulation of cellular biosynthetic process & 0.0 & 0 & -1.9 & 53 \\
\hline
\end{tabular}

Group 2

Subgroup 2A

GO:0051301 cell division

GO:0009825 multidimensional cell growth

GO:0000280 nuclear division

GO:0022403 cell cycle phase

GO:0007000 nucleolus organization

GO:0006275 regulation of DNA replication

GO:0000226 microtubule cytoskeleton organization

GO:0022402 cell cycle process

GO:0000911 cytokinesis by cell plate formation

GO:0040029 regulation of gene expression, epigenetic

GO:0048589 developmental growth

GO:0007017 microtubule-based process

GO:0006996 organelle organization

GO:0007049 cell cycle

GO:0006260 DNA replication

GO:0048507 meristem development

GO:0006261 DNA-dependent DNA replication

GO:0006325 chromatin organization

GO:0006270 DNA replication initiation

GO:0051276 chromosome organization

GO:0008283 cell proliferation

$-1.4$

$-1.5$

$-1.6$

$-1.8$

$-1.9$

$-2.5$

$-2.6$

$-2.9$

$-3.0$

$-3.5$

$-3.6$

$-3.6$

$-3.9$

$-4.0$

$-4.4$

$-4.5$

$-5.0$

$-5.8$

$-6.1$

$-6.7$

$-8.0$

Subgroup 2B

GO:0048522 positive regulation of cellular process

GO:0048518 positive regulation of biological process

GO:0006310 DNA recombination
$-1.6$

$-2.1$

$-2.5$

$\begin{array}{rr}7.4 & 33 \\ 3.0 & 12 \\ 3.8 & 13 \\ 5.3 & 28 \\ 1.8 & 5 \\ 6.4 & 19 \\ 5.3 & 23 \\ 8.7 & 46 \\ 7.4 & 25 \\ 3.3 & 29 \\ 3.7 & 40 \\ 8.1 & 32 \\ 8.0 & 96 \\ 9.7 & 58 \\ 6.1 & 29 \\ 4.5 & 32 \\ 6.1 & 25 \\ 8.7 & 45 \\ 2.7 & 9 \\ 9.5 & 55 \\ 6.2 & 26 \\ & \\ 0.0 & 0 \\ 0.0 & 0 \\ 0.0 & 0\end{array}$

Continued next page 


\begin{tabular}{|c|c|c|c|c|}
\hline \multirow[b]{2}{*}{ GO term } & \multicolumn{2}{|c|}{$3 \mathrm{DAT}$} & \multicolumn{2}{|c|}{$7 \mathrm{DAT}$} \\
\hline & $\mathrm{EV}$ & DEGs (no.) & $\overline{\mathrm{EV}}$ & DEGs (no.) \\
\hline GO:0032774 RNA biosynthetic process & -3.1 & 90 & 0.0 & 0 \\
\hline $\begin{array}{l}\text { GO:0019219 regulation of nucleobase, nucleoside, } \\
\text { nucleotide and nucleic acid metabolic process }\end{array}$ & -4.5 & 94 & 0.0 & 0 \\
\hline GO:0016570 histone modification & -4.9 & 28 & 0.0 & 0 \\
\hline \multicolumn{5}{|l|}{ Subgroup 2C } \\
\hline GO:0071669 plant-type cell wall organization or biogenesis & 0.0 & 0 & 2.2 & 25 \\
\hline GO:0071554 cell wall organization or biogenesis & 0.0 & 0 & 2.3 & 41 \\
\hline GO:0009832 plant-type cell wall biogenesis & 0.0 & 0 & 2.6 & 13 \\
\hline GO:0044238 primary metabolic process & 0.0 & 0 & 3.4 & 317 \\
\hline GO:0035266 meristem growth & 0.0 & 0 & 4.5 & 18 \\
\hline GO:0010389 regulation of $\mathrm{G} 2 / \mathrm{M}$ transition of mitotic cell cycle & 0.0 & 0 & 4.6 & 12 \\
\hline
\end{tabular}

${ }^{\mathrm{z}}$ Enrichment values were the absolute values of $\log _{10}$ false discovery rate-corrected $P$ values based on Fisher's exact test for individual GO terms, where up and downregulated DEGs are indicated as positive and negative values, respectively.

such as cell division (GO:0051301), cell cycle (GO:0007049), cell proliferation (GO:0008283), and DNA replication (GO:0006996) (Table 2).

EFFects of HC ON CYANIDE Metabolism. To investigate the cyanide-related metabolic pathways in which DEGs were involved, KEGG Mapper was used. At 3 DAT, three DEGs encoding the enzymes for the last step of hydrogen cyanide synthesis, including mandelonitrile lyase [MLD (Prupe.1G093600 and Prupe.1G093000)] and catalase [CAT2 (Prupe.5G011400)], were upregulated (Fig. 2), indicating the production of hydrogen cyanide in buds of peach trees treated with $\mathrm{HC}$; however, upstream of mandelonitrile was downregulated. Nevertheless, DEG for a $\beta$-glucosidase $[B G L U 15$ (Prupe.3G111000)], which coverts cyanogenic glycosides prunasin and amygdalin to mandelonitrile, the precursor of cyanide, was downregulated at 3 DAT. In addition, on the same day, a nitrilase [NIT4 (Prupe.6G137600)] that catalyzes the production of aspartate and asparagine from L-3-cyanoalaine, which is the intermediate product of cyanide detoxification by L-3cyanoalaine synthase, was upregulated.

EFFECTS OF HC ON CELlular RESPIRATION. Differentially expressed genes that were involved in respiration, including glycolysis, the tricarboxylic acid (TCA) cycle, and the pentose phosphate pathway (PPP), are presented in Fig. 3. At 3 DAT, DEGs encoding fructose-1,6-bisphosphatase (Prupe.8G028300), triosephosphate isomerase (Prupe.6G076300), 2,3-bisphosphoglycerate-independent phosphoglycerate mutase (Prupe.6G300000), and enolase (Prupe.2G281900) were upregulated; the DEG encoding pyruvate kinase (Prupe.8G151100), during the last step of glycolysis and during which ATP is produced, was downregulated in buds of HCtreated trees (Fig. 3A). Interestingly, DEGs encoding enzymes in the PPP, such as glucose-6-phosphate dehydrogenase [G6PD (Prupe.6G307600)] and transketolase (Prupe.2G143600), were upregulated at 3 DAT, indicating an increase in NADPH in the buds of trees treated with HC. At 7 DAT, the DEG encoding a pyruvate kinase (Prupe.7G120700) for the ATP-producing step of glycolysis was upregulated
(Fig. 3B). Moreover, DEGs that encode enzymes for the TCA cycle, including pyruvate dehydrogenase (Prupe.1G262100), dihydrolipoamide acetyltransferase (Prupe.2G204600), isocitrate dehydrogenase (Prupe.3G288200), and phosphoenolpyruvate carboxykinase (Prupe.6G210900), were upregulated at 7 DAT.

WRKY AND EXPANSIN TRANSCRIPTION FACTOR FAMILIES. Several genes encoding transcription factors (TFs) were differentially expressed as a result of $\mathrm{HC}$ application at both 3 and 7 DAT (Supplemental Table 3). WRKY is a TF family of particular interest because many members of this family have been reported to be related to not only pathogen responses but also stress responses (Babitha et al., 2013; Chen et al., 2012). At 3 DAT, DEGs encoding WRKY6, WRKY28, WRKY40, and WRKY75 were upregulated, whereas that encoding WRKY12 was downregulated (Table 4). At 7 DAT, DEGs encoding WRKY7 and WRKY57 were upregulated and DEGs encoding WRKY22 were downregulated. The application of $\mathrm{HC}$ also had effects on the expression of the genes encoding expansins (EXPs), a family of nonenzymatic proteins in plant cell walls (Cosgrove, 2000). At 3 DAT, eight DEGs encoding EXPs were downregulated and two were upregulated (Table 5). Nonetheless, DEGs for EXP3, EXPA1, EXPA8, EXPA15, and EXPB2 ( 5 out of 6 DEGs) were upregulated in buds of HC-treated trees at 7 DAT.

ReLATIVE GeNe EXPRESSION anALySIS. Despite being differentially expressed at 3 DAT based on the RNA-seq analysis, the levels of FDH, MSD1, GSTU8, GSTU19, PMSR1, and NQR expression quantified using qPCR were not significantly greater in buds of HC-treated trees compared with nontreated controls on any sampling day (Supplemental Fig. 2). This discrepancy between the results of qPCR and RNA-seq analyses could be attributed to the different principles of the two technologies. Nevertheless, there was a positive and significant correlation between relative expression levels (obtained by qPCR analysis) and fold changes (obtained by RNA-seq analysis) for these six genes $(r=0.80 ; P=0.050)$, indicating that the RNA-seq data are valid. 
Table 3. Antioxidants and related enzymes encoded by differentially expressed genes (DEGs) with $\log _{2}$ fold changes (LFC) in buds of 'TropicBeauty' peach trees treated with $1 \%(\mathrm{v} / \mathrm{v})$ hydrogen cyanamide (HC) compared with nontreated control trees at $3 \mathrm{~d}$ after treatment.

\begin{tabular}{|c|c|c|c|}
\hline \multicolumn{3}{|c|}{ Arabidopsis thaliana } & LFC \\
\hline \multicolumn{4}{|c|}{ Superoxide dismutase (superoxide $+2 \mathrm{H}^{+} \rightarrow \mathrm{H}_{2} \mathrm{O}_{2}+\mathrm{O}_{2}$ ) } \\
\hline Prupe.2G262400 & AT3G10920 & Manganese superoxide dismutase 1 & 1.0 \\
\hline Prupe.2G269400 & AT5G51100 & Iron superoxide dismutase 2 & -1.8 \\
\hline \multicolumn{4}{|c|}{ Catalase $\left(2 \mathrm{H}_{2} \mathrm{O}_{2} \rightarrow 2 \mathrm{H}_{2} \mathrm{O}+\mathrm{O}_{2}\right)$} \\
\hline Prupe.5G011400 & AT4G35090 & Catalase 2 & 1.9 \\
\hline \multicolumn{4}{|c|}{ Peroxidase $\left(2 \mathrm{H}_{2} \mathrm{O}_{2}+\mathrm{RH}_{2} \rightarrow 2 \mathrm{H}_{2} \mathrm{O}+\mathrm{R}\right)$} \\
\hline Prupe.6G289400 & AT5G05340 & Peroxidase superfamily protein & 3.2 \\
\hline Prupe.8G038900 & AT1G71695 & Peroxidase superfamily protein & 1.5 \\
\hline Prupe.4G021100 & AT4G21960 & Peroxidase superfamily protein & -2.0 \\
\hline Prupe.1G397400 & AT2G24800 & Peroxidase superfamily protein & -2.6 \\
\hline Prupe.6G091600 & AT1G07890 & Ascorbate peroxidase 1 & 1.0 \\
\hline Prupe.6G155100 & AT5G21105 & Plant L-ascorbate oxidase & -3.4 \\
\hline \multicolumn{4}{|c|}{ Thioredoxin (R-S-S-R $\rightarrow 2$ R-SH) } \\
\hline Prupe.1G140400 & AT5G18600 & Thioredoxin superfamily protein & 3.7 \\
\hline Prupe.8G126900 & AT2G15570 & Thioredoxin superfamily protein & 2.1 \\
\hline Prupe.5G170800 & AT5G63030 & Thioredoxin superfamily protein & -1.6 \\
\hline Prupe.1G265600 & AT5G39950 & Thioredoxin 2 & -2.1 \\
\hline \multicolumn{4}{|c|}{ Enzymes maintaining the scavenger function of ascorbate } \\
\hline Prupe.3G286700 & AT3G27820 & Monodehydroascorbate reductase 4 & 1.3 \\
\hline Prupe.2G227100 & AT1G75270 & Dehydroascorbate reductase 2 & -1.2 \\
\hline \multicolumn{4}{|c|}{ Enzymes maintaining the scavenger function of glutathione } \\
\hline Prupe.6G244700 & AT5G02790 & Glutathione S-transferase family protein & 2.0 \\
\hline Prupe.1G264700 & AT1G10370 & Glutathione S-transferase family protein & -1.4 \\
\hline Prupe.8G256900 & AT2G47730 & Glutathione S-transferase PHI 8 & 4.4 \\
\hline Prupe.8G210800 & AT2G29420 & Glutathione S-transferase TAU 7 & 3.8 \\
\hline Prupe.1G526000 & AT2G29420 & Glutathione S-transferase TAU 7 & 2.9 \\
\hline Prupe.2G101600 & AT2G29420 & Glutathione S-transferase TAU 7 & 2.5 \\
\hline Prupe.1G055000 & AT2G29420 & Glutathione S-transferase TAU 7 & 1.7 \\
\hline Prupe.8G210700 & AT3G09270 & Glutathione S-transferase TAU 8 & 3.8 \\
\hline Prupe.6G264900 & AT3G09270 & Glutathione S-transferase TAU 8 & 3.5 \\
\hline Prupe.5G228000 & AT3G09270 & Glutathione S-transferase TAU 8 & 2.9 \\
\hline Prupe.4G147400 & AT1G78380 & Glutathione S-transferase TAU 19 & 5.7 \\
\hline Prupe.4G146800 & AT1G78380 & Glutathione S-transferase TAU 19 & 2.9 \\
\hline Prupe.4G146400 & AT1G78380 & Glutathione S-transferase TAU 19 & 2.3 \\
\hline Prupe.4G147000 & AT1G78380 & Glutathione S-transferase TAU 19 & 2.1 \\
\hline Prupe.4G147200 & AT1G78380 & Glutathione S-transferase TAU 19 & 1.1 \\
\hline Prupe.4G147100 & AT1G78380 & Glutathione S-transferase TAU 19 & 1.1 \\
\hline Prupe.4G146000 & AT1G78380 & Glutathione S-transferase TAU 19 & -2.3 \\
\hline Prupe.3G165500 & AT1G65820 & Microsomal glutathione s-transferase & 1.1 \\
\hline \multicolumn{4}{|c|}{ Other enzymes with reducing activity } \\
\hline Prupe.5G161000 & AT5G61640 & Peptide methionine sulfoxide reductase & 1.3 \\
\hline Prupe.2G038400 & AT3G27890 & NADPH:quinone oxidoreductase & 2.3 \\
\hline
\end{tabular}

\section{Discussion}

For 'TropicBeauty' peach, maximum budbreak occurred at 32 DAT in trees treated with $\mathrm{HC}$ and at $74 \mathrm{DAT}$ in nontreated trees (Fig. 1). The results reported herein demonstrated that the application of HC in mid-December advanced budbreak by 6 weeks, consistent with results reported previously (George et al., 1992; Olmstead, 2014; Yamane et al., 2011). Although the levels of DAM1,DAM5, and DAM6 transcripts in buds were not significantly affected within $7 \mathrm{~d}$ after $\mathrm{HC}$ application (Supplemental Fig. 3), the results cannot exclude the possibility that the repression of $D A M$ expression by $\mathrm{HC}$ may occur after $7 \mathrm{~d}$. This possibility is supported by the results demonstrating a decrease in DAM5 and $D A M 6$ expression levels in vegetative peach buds in response to cyanamide at 2 and 4 weeks after treatment, respectively (Yamane et al., 2011).

At 3 DAT, 4 weeks before maximum budbreak, metabolic processes involving nitrile lyases and nitrilases were upregulated (Table 1). In plants, hydrogen cyanide can be generated through cyanogenesis, which is the hydrolysis of cyanogenic glycosides that requires cyanogenic $\beta$-glucosidase and nitrile lyase (Gleadow and Møller, 2014; Morant et al., 2008). For sweet cherry, genes for both enzymes had greater transcript levels in buds from the branches sprayed with HC (followed by accelerated budbreak) than those from the nontreated branches (Ionescu et al., 2017b). Consistently, for buds of HC-treated trees in this study, in addition to $M L D$, the DEG encoding CAT2, which is involved in cyanide synthesis via a different pathway (Ionescu et al., 2017a; Shirota et al., 1987), was upregulated at 3 DAT (Fig. 2), indicating that exogenous $\mathrm{HC}$ application caused an osten-

The expressions of DAM1, DAM5, and DAM6 did not change over the sampling time in buds of nontreated trees (Supplemental Fig. 3). The application of HC, despite advancing budbreak by 6 weeks, did not result in significant differences in the levels of DAM1,DAM5, and DAM6 expression within $7 \mathrm{~d}$ following the treatment compared with the nontreated control. There was no significant difference in the expression levels of floral organ identity genes $A P 1, P I, A G$, and $F B P 9$ on any sampling day in HC-treated and nontreated trees (Supplemental Fig. 3). It should also be noted that none of these seven dormancy-related or flowering-related genes was differentially expressed in HC-treated and nontreated trees at either 3 or 7 DAT based on RNA-seq analysis. sible increase in hydrogen cyanide in buds at 3 DAT. In plants, toxic cyanide produced endogenously is eradicated primarily by $\beta$-cyanoalanine synthase ( $\beta$-CAS), resulting in L-3-cyanoalaine, which can be further hydrolyzed to aspartate and asparagine by NIT4 as a part of the detoxification system (Piotrowski et al., 2001; Siegien and Bogatek, 2006). In the current study, although $\beta-C A S$ was not differentially expressed, NIT4 was upregulated in buds of HC-treated trees at 3 DAT (Fig. 2), suggesting the need for hydrogen cyanide detoxification in response to its production in buds triggered by exogenous $\mathrm{HC}$ application.

Hydrogen cyanide interrupts cellular respiration by disrupting the electron transport pathway through binding to cyto- 


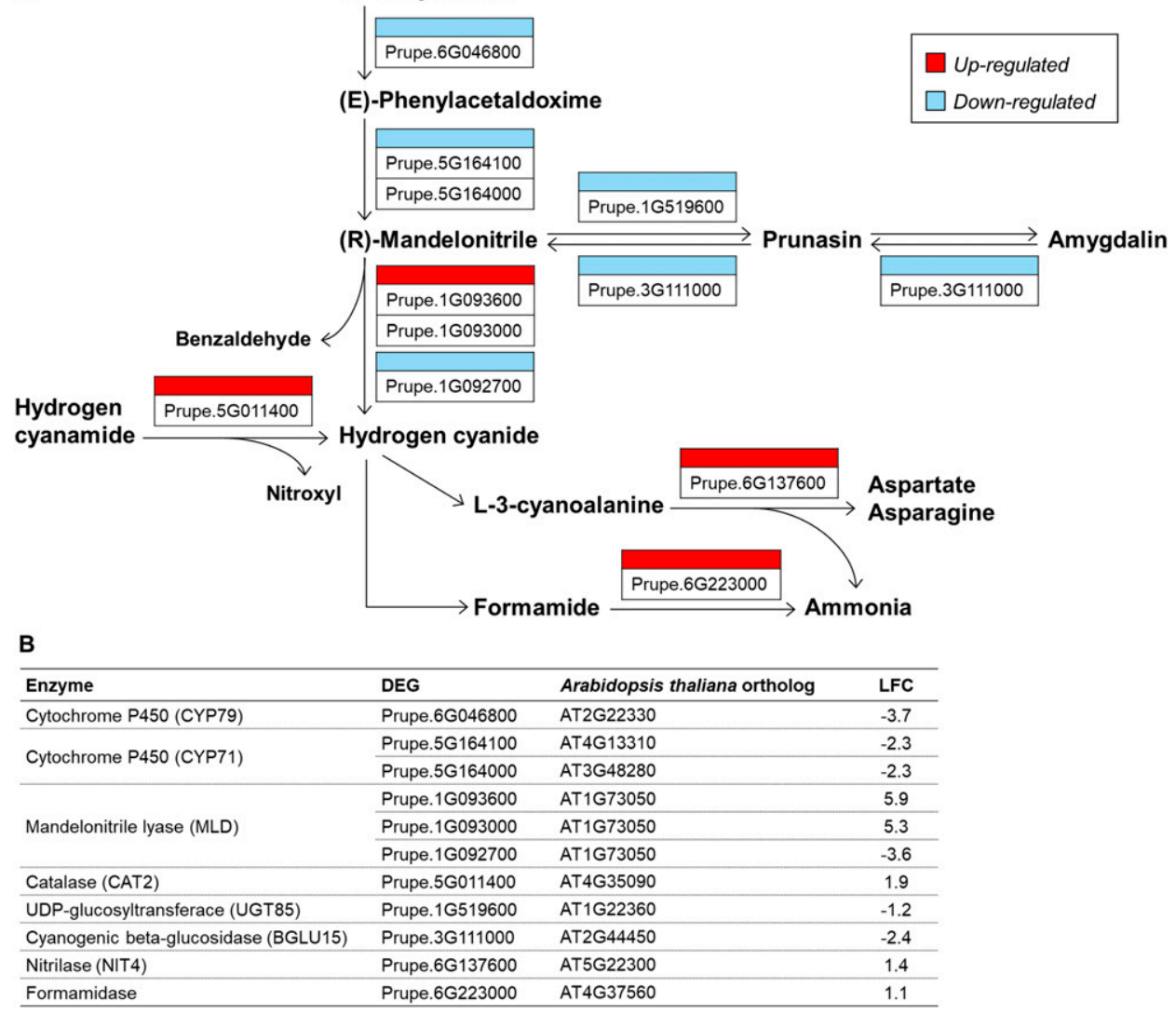

Fig. 2. Overview of hydrogen cyanide-related metabolic pathways in which enzymes were encoded by the differentially expressed genes (DEGs), with red and blue indicating upregulated and downregulated DEGs, respectively, in buds of 'TropicBeauty' peach trees treated with $1 \%(\mathrm{v} / \mathrm{v})$ hydrogen cyanamide compared with nontreated control trees at $3 \mathrm{~d}$ after treatment (DAT) (A). A list of these enzymes, DEGs, putative Arabidopsis thaliana orthologs, and $\log _{2}$ fold change (LFC) at 3 DAT (B) is provided.
(GO:0070482), ROS metabolic process (GO:0006800), and regulation of ROS metabolic process (GO:0080010) were upregulated in buds of HC-treated trees at 3 DAT (Table 2), suggesting an increase in ROS generation under the hypoxic conditions imposed by HC. In addition, on the same day, WRKY6, WRKY28, WRKY40, and WRKY75, which belong to the WRKY TF family and have a role in oxidative stress (Bakshi and Oelmüller, 2014; Chen et al., 2012; Scarpeci et al., 2008), were upregulated (Table 4). Among them, expressions of WRKY 6 and WRKY75 have been demonstrated to be increased in response to hydrogen peroxide in arabidopsis (Arabidopsis thaliana) (Bakshi and Oelmüller, 2014), lending further support to the possibility that HCtriggered oxidative stress leads to the accumulation of ROS in peach buds.

The DEGs for lipid oxidation (GO:0034440) were upregulated in buds of HC-treated trees at 3 DAT (Table 2). It is of relevance that oxidation or peroxidation of lipids, which are generally accepted as a hallmark of oxidative stress, causes the loss of membrane integrity and cell damage (Demidchik, 2015; Farmer and Mueller, 2013). The results of the current research demonstrated that DEGs for the GO terms

chrome c oxidase (COX) (Antonini et al., 1971; Bendall and Bonner, 1971; Cooper and Brown, 2008). In grape, exogenous HC application, which accelerated budbreak, also inhibited the uptake of oxygen in mitochondria (Pérez et al., 2009). Interestingly, DEGs encoding an alternative oxidase, AOXIa (Prupe.5G018700), which has a similar function as COX in the electron transport chain of respiration but is insensitive to cyanide toxicity (Vanlerberghe and McIntosh, 1997), and an alcohol dehydrogenase, Prupe.8G018300, which is involved in fermentation (anaerobic respiration) (Or et al., 2000; Perata and Alpi, 1993), were upregulated in buds at 3 DAT (Fig. 3C). Taken together, the results of the current research suggested that in peach buds, the application of $\mathrm{HC}$ and $\mathrm{HC}$-induced endogenous hydrogen cyanide triggered similar metabolic responses to hypoxia as a result of dysfunctional mitochondrial respiration.

Under hypoxia, the production of ROS increases (Blokhina and Fagerstedt, 2010; Turrens, 2003); furthermore, accumulations of hydrogen peroxide, superoxide, and nitric oxide were observed in grapevine buds after $12 \mathrm{~h}$ of $\mathrm{HC}$ treatment that induced budbreak in the summer (Sudawan et al., 2016). For peach, the ROS content in buds in relation to the release of endodormancy by either natural chill hours or exogenous treatments has never been investigated. Nonetheless, in this study, DEGs for oxidative stress (GO:0006979), response to hypoxia (GO:0001666), response to oxygen levels related to cell cycle, growth, and development were downregulated in buds of HC-treated trees at 3 DAT (Tables 1 and 2 ), thus providing evidence that normal cell activities in buds were repressed by $\mathrm{HC}$-induced oxidative stress within only $3 \mathrm{~d}$ after application. For HC-treated trees, bud $F D H$ was differentially expressed at 3 DAT according to the RNA-seq analysis. In arabidopsis, $F D H$ transcripts were increased under stress conditions such as hypoxia (Li et al., 2001), and this gene was reported to have a role in cell death and defense responses to bacterial pathogens in pepper [Capsicum annuum (Choi et al., 2014)]. Consistently, the current results indicating the upregulation of programmed cell death (GO:0012501), cell death (GO:0008219), and death (GO:0016265) at 3 DAT (Table 2) further suggest a detrimental effect of $\mathrm{HC}$ on cell proliferation. Nevertheless, in many plants, layers of dead cells shield the living tissues from harmful factors under stress conditions (Demidchik et al., 2014; Demidchik, 2015), suggesting that a strategy for survival under oxidative stress may be triggered by $\mathrm{HC}$ in buds of peach trees.

Glucose 6-phosphate dehydrogenase is the first rate-limiting enzyme of the PPP, and its expression has been reported to be related to the defense against oxidative stress (Boada et al., 2000; Couée et al., 2006; Salvemini et al., 1999). In grapevine, an increase in $G 6 P D$ transcripts was observed at 1,2, and $4 \mathrm{~d}$ after HC treatment (Pérez et al., 2009). It is of interest that G6PD and the DEG encoding a transketolase were upregulated in the buds of HC-treated peach trees at 3 DAT, but not at 7 


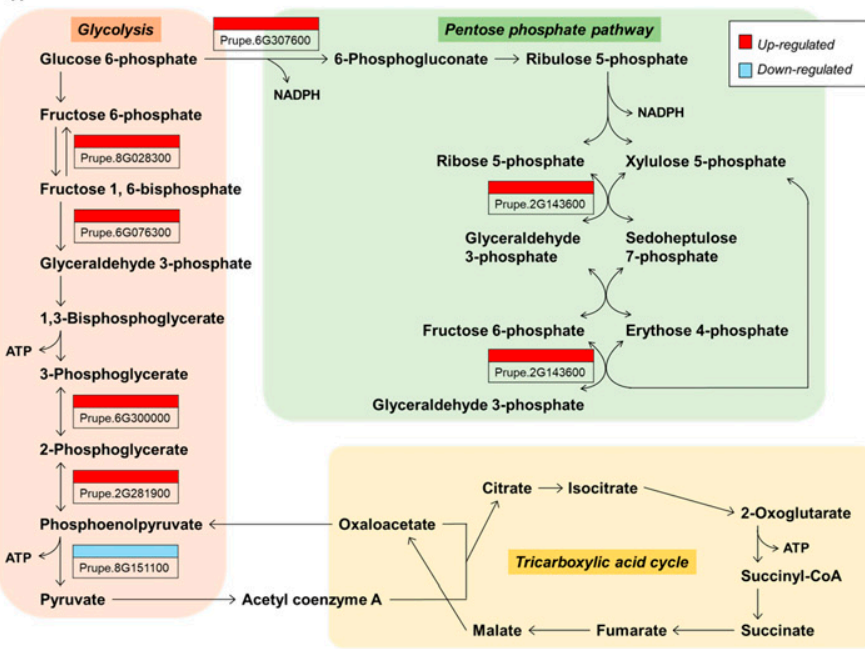

C

\begin{tabular}{llllll}
\hline & \multirow{2}{*}{ Enzyme } & \multirow{2}{*}{$\begin{array}{l}\text { Arabidopsis } \\
\text { thaliana ortholog }\end{array}$} & \multicolumn{2}{c}{ LFC } \\
\cline { 3 - 6 } & & 3 DAT & 7 DAT \\
\hline Fructose-1,6-bisphosphatase & Prupe.8G028300 & AT1G43670 & 1.4 & - \\
\hline Triosephosphate isomerase & Prupe.6G076300 & AT3G55440 & 1.0 & - \\
\hline 2,3 -bisphosphoglycerate-indepnedent phosphoglycerate mutase & Prupe.6G300000 & AT3G08590 & 1.1 & - \\
\hline Enolase & Prupe.2G281900 & AT2G36530 & 2.2 & - \\
\hline Pyruvate kinase & Prupe.8G151100 & AT3G22960 & -1.1 & - \\
\hline Glucose-6-phosphate dehydrogenase & Prupe.6G307600 & AT1G09420 & 1.7 & - \\
\hline Transketolase & Prupe.2G143600 & AT2G45290 & 1.1 & - \\
\hline Alternative oxidase 1A & Prupe.5G018700 & AT3G22370 & 1.8 & - \\
\hline Alcohol dehydrogenase & Prupe.8G018300 & AT1G77120 & 1.7 & - \\
\hline Alcohol dehydrogenase & Prupe.8G018400 & AT1G77120 & -3.0 & - \\
\hline Phosphoglycerate mutase & Prupe.7G136800 & AT3G50520 & - & 2.0 \\
\hline Pyruvate kinase & Prupe.7G120700 & AT3G52990 & - & 1.7 \\
\hline Pyruvate dehydrogenase complex E1 alpha subunit & Prupe.1G262100 & AT1G59900 & - & 1.3 \\
\hline Dihydrolipoamide acetyltransferase & Prupe.2G204600 & AT3G52200 & - & 1.0 \\
\hline Isocitrate/isopropylmalate dehydrogenase & Prupe.3G288200 & AT5G14590 & - & 1.1 \\
\hline Phosphoenolpyruvate carboxykinase & Prupe.6G210900 & AT4G37870 & - & 1.6 \\
\hline Ribose-5-phosphate isomerase & Prupe.1G322200 & AT2G01290 & - & 1.2 \\
\hline & & & & & \\
\hline
\end{tabular}

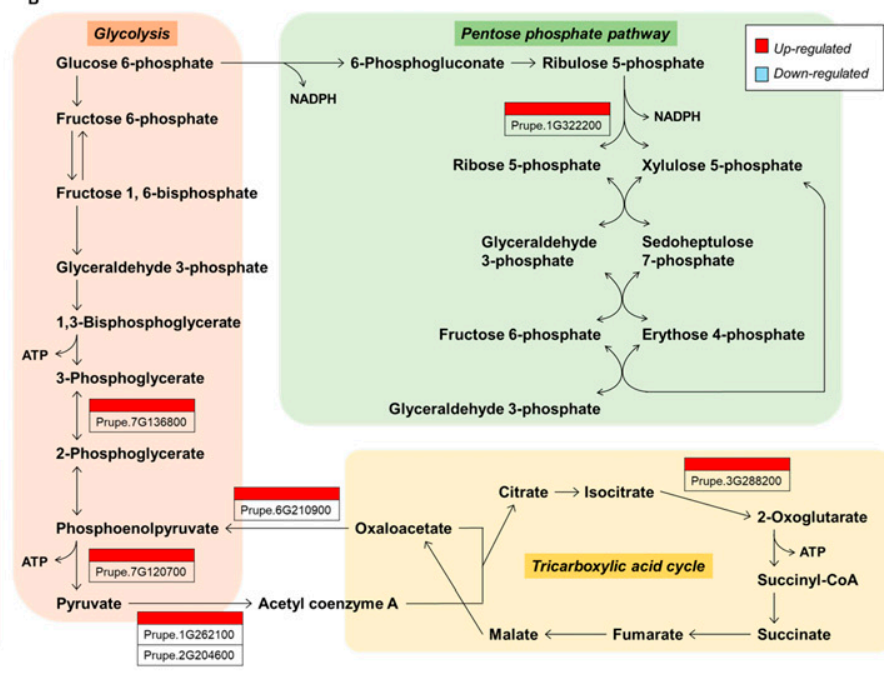

Fig. 3. Overview of glycolysis (highlighted in orange), tricarboxylic acid cycle (highlighted in yellow), and pentose phosphate pathway (highlighted in green) in which enzymes were encoded by the differentially expressed genes (DEGs), with red and blue indicating upregulated and downregulated DEGs, respectively, in buds of 'TropicBeauty' peach trees treated with $1 \%(\mathrm{v} / \mathrm{v})$ hydrogen cyanamide compared with nontreated control trees at $3 \mathrm{~d}$ after treatment (DAT) (A) and 7 DAT (B). A list of these enzymes, DEGs, putative Arabidopsis thaliana orthologs, and $\log _{2}$ fold change (LFC) at 3 and 7 DAT (C) is provided.

Table 4. Members of WRKY transcription factor family encoded by differentially expressed genes (DEGs) with $\log _{2}$ fold changes (LFC) in buds of 'TropicBeauty' peach trees treated with $1 \%(\mathrm{v} / \mathrm{v})$ hydrogen cyanamide compared with nontreated trees at 3 and $7 \mathrm{~d}$ after treatment (DAT).

\begin{tabular}{lcccc}
\hline WRKY member & DEG & Arabidopsis thaliana ortholog & LFC at 3 DAT & LFC at 7 DAT \\
\hline WRKY6 & Prupe.4G017600 & AT1G62300 & 1.3 & - \\
WRKY7 & Prupe.1G431100 & AT4G24240 & - & 1.7 \\
WRKY12 & Prupe.2G133800 & AT2G44745 & -2.0 & - \\
WRKY22 & Prupe.2G177400 & AT4G01250 & - & -1.7 \\
WRKY28 & Prupe.3G174300 & AT4G18170 & 2.7 & - \\
WRKY40 & Prupe.3G098100 & AT1G80840 & 1.9 & - \\
WRKY57 & Prupe.1G283500 & AT1G69310 & - & 2.5 \\
WRKY75 & Prupe.3G308200 & AT5G13080 & 4.2 & -
\end{tabular}

DAT (Fig. 3), indicating a temporary induction of the PPP, which is a response to oxidative stress documented in arabidopsis (Baxter et al., 2007). Cytosolic PPP generates NADPH, which has a pivotal role in removing ROS in the scavenging systems (Beauvieux et al., 2018; May et al., 1998; Mittler, 2002; Pandolfi et al., 1995). Therefore, for buds of peach trees treated with $\mathrm{HC}$ in this study, it is likely that, after the induction of the PPP, the reducing power was enhanced in the form of NADPH to surmount oxidative stress.

At 3 DAT, 26 DEGs encoding the antioxidants and enzymes maintaining their scavenging activities were upregulated in buds of HC-treated trees according to the RNA-seq results
(Table 3). Among them were superoxide dismutase, catalase, peroxidase superfamily protein, ascorbate peroxidase, thioredoxin, dehydroascorbate reductase, and GST, of which the transcript levels and enzymatic activity have been reported to be correlated with the release of bud dormancy in response to dormancy-breaking treatments for apricot [Prunus armeniaca (Scalabrelli et al., 1991; Viti et al., 2012)], grapevines (Halaly et al., 2008), japanese apricot [Prunus mume (Zhuang et al., 2013)], and japanese pear [Pyrus pyrifolia (Bai et al., 2013)]. As many as 16 DEGs for GST were upregulated at 3 DAT (Table 3), consistent with the results of the MapMan analysis indicating the upregulation of the metabolic process involving GST (BIN 26.9) at 3 DAT (Table 1). For grapevine, HC treatment used to break bud dormancy resulted in an increase in GST transcript levels at 2 and 4 DAT, respectively, compared with the nontreated control (Halaly et al., 2008; Keilin et al., 2007), in agreement with the results presented here. Taken together, the results of the current research suggest that within $3 \mathrm{~d}$ after the $\mathrm{HC}$ application, the detoxification mechanism against ROS 
Table 5. Members of expansin (EXP) transcription factor family encoded by differentially expressed genes (DEGs) with $\log _{2}$ fold changes (LFC) in buds of 'TropicBeauty' peach trees treated with $1 \%$ (v/v) hydrogen cyanamide compared with nontreated trees at 3 and $7 \mathrm{~d}$ after treatment (DAT).

\begin{tabular}{lcccc}
\hline & & & LFC at 3 & LFC at 7 \\
EXP member & DEG & Arabidopsis thaliana ortholog & DAT & DAT \\
\hline EXP3 & Prupe.6G256500 & AT2G37640 & -1.1 & 2.3 \\
EXPA1 & Prupe.1G276700 & AT1G69530 & -2.1 & 1.9 \\
EXPA4 & Prupe.6G075100 & AT2G39700 & -1.3 & - \\
EXPA8 & Prupe.2G263600 & AT2G40610 & -2.6 & 2.7 \\
EXPA8 & Prupe.6G042000 & AT2G40610 & -3.0 & - \\
EXPA15 & Prupe.3G265800 & AT2G03090 & -1.7 & 2.0 \\
EXPB2 & Prupe.2G136500 & AT1G65680 & -1.4 & - \\
EXPB2 & Prupe.2G274400 & AT1G65680 & - & 1.7 \\
EXPB3 & Prupe.3G155600 & AT4G28250 & -2.3 & - \\
EXP-like B1 & Prupe.5G057900 & AT4G17030 & 4.9 & -2.1 \\
EXP-like B1 & Prupe.5G047300 & AT4G17030 & 1.6 & - \\
\hline
\end{tabular}

degree of the opening of flower buds of four o'clock flower [Mirabilis jalapa (Gookin et al., 2003)], it is possible that the upregulation of bud EXP expression by $\mathrm{HC}$ at 7 DAT resulted in the swelling and burst of buds 3 weeks later (at 32 DAT) in peach. For HC-treated grapevine that had a greater budbreak rate than the nontreated control, ROS were accumulated in buds at $12 \mathrm{~h}$ after treatment before the upregulation of EXP genes (Sudawan et al., 2016). It is still unclear if ROS induce the modification of cell wall structures that results in budbreak in deciduous trees. Nevertheless, it has been reported that ROS have a role in

triggered by exogenous $\mathrm{HC}$ application has already been activated in peach buds.

At 7 DAT, most of the GO terms associated with stress responses were no longer statistically significant, and DEGs for the response to oxidative stress (GO:0006979) were downregulated in buds of HC-treated trees (group 1 in Table 2), indicating that $\mathrm{HC}$-induced oxidative stress was transient and offset within $7 \mathrm{~d}$ following the application, consistent with previous studies of grapevine (Or et al., 2000; Pérez et al., 2009; Sudawan et al., 2016). The results presented in this report demonstrated that DEGs encoding enzymes during the last step in glycolysis and those for TCA cycle were upregulated, and that G6PD for PPP was not differentially expressed in buds of HCtreated trees at 7 DAT (Fig. 3), suggesting that the carbon substrate is oxidized via glycolysis and subsequently enters the TCA cycle for efficient energy production. Based on the MapMan enrichment analysis, DNA synthesis (BIN 29.4.1) and cell organization (BIN 31.1) were upregulated at 7 DAT (Table 1). Similarly, for DEGs of the GO terms for cell development and proliferation, there was a shift from downregulation at 3 DAT to upregulation at 7 DAT (group 2A in Table 2). These results provided evidence that in peach buds, growth and development did not resume after the initial inhibition by $\mathrm{HC}$; instead, they were markedly enhanced within 1 week after HC application. Accordingly, it is speculated that the increased ATP production as a result of increased aerobic respiration is used to supply the metabolic and physiological processes for cell growth and tissue development in preparation for budbreak. In support of this possibility, Bonhomme et al. (2000) reported that in peach buds, the ATP concentration was very low during the dormancy phase and increased rapidly when dormancy was released following the fulfillment of the chilling requirement.

Because cell walls loosen to allow the expansion of flower buds of rose (Rosa hybrida) upon opening (Yamada et al., 2009), the gene sets associated with cell wall loosening were of particular interest in this study. The results of both MapMan and GO enrichment analyses demonstrated the upregulation of processes involving the degradation and modification of the cell wall at 7 DAT (Tables 1 and 2). On the same day, DEGs encoding five EXPs, which are proteins responsible for acidinduced wall extension without any enzymatic activity (Cosgrove, 2000), were also upregulated, including EXP3, EXPA1, EXPA8, EXPA15, and EXLB1 (Table 5). Given that bud $E X P$ transcript levels were positively correlated with the wall loosening of growing tissues in the roots of maize [Zea mays (Liszkay et al., 2004)], roots of arabidopsis (Joo et al., 2001), and seeds of cress (Lepidium sativum) during germination (Müller et al., 2009).

The results of this study provide evidence that, for low-chill 'TropicBeauty' peach, exogenous HC application causes transient oxidative stress that likely induces the release of endodormancy, in agreement with the currently hypothesized mode of $\mathrm{HC}$ action for other fruit crops. The results also strongly suggest that HC-triggered oxidative stress enhances cell development and bud enlargement, ultimately leading to earlier budbreak than that in nontreated peach trees. Nonetheless, the underlying mechanism of the promotion of dormancy release by oxidative stress remains to be determined. Recently, ROS have been shown to serve as signal molecules that are essential for proper cellular function and normal physiological processes, which is in contrast to their traditional association with cell damage and death in relation to stress (Mittler, 2017; Mittler et al., 2004, 2011; Reczek and Chandel, 2015). Therefore, the results of the current research suggesting the generation of ROS under oxidative stress in buds of HC-treated peach trees at 3 DAT, along with the increased ROS levels in japanese pear and grapevine buds before budbreak in response to HC application (Kuroda et al., 2002; Sudawan et al., 2016), indicate the possibility that $\mathrm{HC}$ advances budbreak in peach by inducing the production and accumulation of ROS that mediate the regulation of physiological events such as differentiation of the meristem and cell wall expansion. To address this, further studies of HC-treated peach trees including an assessment of the expression and activity of the enzymes involved in ROS generation and turnover, such as respiratory burst oxidase homologs, glutaredoxins thioredoxins, and peroxiredoxins (Kobayashi et al., 2007; Mittler, 2017; Ogasawara et al., 2008; Rouhier, 2010), are necessary, as is close monitoring of ROS contents in buds.

\section{Literature Cited}

Antonini, E., M. Brunori, C. Greenwood, B.G. Malmström, and G.C. Rotilio. 1971. The interaction of cyanide with cytochrome oxidase. Eur. J. Biochem. 23:396-400.

Babitha, K.C., S.V. Ramu, V. Pruthvi, P. Mahesh, K.N. Nataraja, and M. Udayakumar. 2013. Co-expression of AtbHLH17 and AtWRKY28 confers resistance to abiotic stress in Arabidopsis. Transgenic Res. 22:327-341. 
Bai, S., T. Saito, D. Sakamoto, A. Ito, H. Fujii, and T. Moriguchi. 2013. Transcriptome analysis of japanese pear (Pyrus pyrifolia Nakai) flower buds transitioning through endodormancy. Plant Cell Physiol. 54:1132-1151.

Bakshi, M. and R. Oelmüller. 2014. WRKY transcription factors. Plant Signal. Behav. 9:e27700.

Barrett, T., S.E. Wilhite, P. Ledoux, C. Evangelista, I.F. Kim, M. Tomashevsky, K.A. Marshall, K.H. Phillippy, P.M. Sherman, M. Holko, A. Yefanov, H. Lee, N. Zhang, C.L. Robertson, N. Serova, S. Davis, and A. Soboleva. 2013. NCBI GEO: Archive for functional genomics data sets-update. Nucleic Acids Res. 41:D991-D995.

Baxter, C.J., H. Redestig, N. Schauer, D. Repsilber, K.R. Patil, J. Nielsen, J. Selbig, A. Liu, A.R. Fernie, and L.J. Sweetlove. 2007. The metabolic response of heterotrophic Arabidopsis cells to oxidative stress. Plant Physiol. 143:312-325.

Beauvieux, R., B. Wenden, and E. Dirlewanger. 2018. Bud dormancy in perennial fruit tree species: A pivotal role for oxidative cues. Front. Plant Sci. 9:657.

Bendall, D.S. and W.D. Bonner. 1971. Cyanide-insensitive respiration in plant mitochondria. Plant Physiol. 47:236-245.

Bielenberg, D.G., Y. Wang, Z. Li, T. Zhebentyayeva, S. Fan, G.L. Reighard, R. Scorza, and A.G. Abbott. 2008. Sequencing and annotation of the ever-growing locus in peach [Prunus persica (L.) Batsch] reveals a cluster of six MADS-box transcription factors as candidate genes for regulation of terminal bud formation. Tree Genet. Genomes 4:495-507.

Blokhina, O. and K.V. Fagerstedt. 2010. Oxidative metabolism, ROS and NO under oxygen deprivation. Plant Physiol. Biochem. 48:359373.

Boada, J., T. Roig, X. Perez, A. Gamez, R. Bartrons, M. Cascante, and J. Bermúdez. 2000. Cells overexpressing fructose-2,6-bisphosphatase showed enhanced pentose phosphate pathway flux and resistance to oxidative stress. FEBS Lett. 480:261-264.

Bonhomme, M., R. Rageau, and M. Gendraud. 2000. Influence of temperature on the dynamics of ATP, ADP and non-adenylic triphosphate nucleotides in vegetative and floral peach buds during dormancy. Tree Physiol. 20:615-621.

Bound, S.A. and K.M. Jones. 2004. Hydrogen cyanamide impacts on flowering, crop load, and fruit quality of red 'Fuji' apple (Malus domestica). N. Z. J. Crop Hort. Sci. 32:227-234.

Chen, L., Y. Song, S. Li, L. Zhang, C. Zou, and D. Yu. 2012. The role of WRKY transcription factors in plant abiotic stresses. Biochim. Biophys. Acta 1819:120-128.

Choi, D.S., N.H. Kim, and B.K. Hwang. 2014. Pepper mitochondrial FORMATE DEHYDROGENASE1 regulates cell death and defense responses against bacterial pathogens. Plant Physiol. 166:12981311.

Considine, M.J. and J.A. Considine. 2016. On the language and physiology of dormancy and quiescence in plants. J. Expt. Bot. 67:3189-3203.

Cooper, C.E. and G.C. Brown. 2008. The inhibition of mitochondrial cytochrome oxidase by the gases carbon monoxide, nitric oxide, hydrogen cyanide and hydrogen sulfide: Chemical mechanism and physiological significance. J. Bioenerg. Biomembr. 40:533.

Cosgrove, D.J. 2000. Expansive growth of plant cell walls. Plant Physiol. Biochem. 38:109-124.

Couée, I., C. Sulmon, G. Gouesbet, and A. El Amrani. 2006. Involvement of soluble sugars in reactive oxygen species balance and responses to oxidative stress in plants. J. Expt. Bot. 57:449-459.

Demidchik, V., D. Straltsova, S.S. Medvedev, G.A. Pozhvanov, A. Sokolik, and V. Yurin. 2014. Stress-induced electrolyte leakage: The role of $\mathrm{K}^{+}$-permeable channels and involvement in programmed cell death and metabolic adjustment. J. Expt. Bot. 65:1259-1270.

Demidchik, V. 2015. Mechanisms of oxidative stress in plants: From classical chemistry to cell biology. Environ. Exp. Bot. 109:212-228. Dennis, F.G. 2003. Problems in standardizing methods for evaluating the chilling requirements for the breaking of dormancy in buds of woody plants. HortScience 38:347-350.
Edgar, R., M. Domrachev, and A.E. Lash. 2002. Gene expression omnibus: NCBI gene expression and hybridization array data repository. Nucleic Acids Res. 30:207-210.

Farmer, E.E. and M.J. Mueller. 2013. ROS-mediated lipid peroxidation and RES-activated signaling. Annu. Rev. Plant Biol. 64:429450.

George, A.P., J. Lloyd, and R.J. Nissen. 1992. Effects of hydrogen cyanamide, paclobutrazol and pruning date on dormancy release of the low chill peach cultivar Flordaprince in subtropical Australia. Austral. J. Expt. Agr. 32:89-95.

Gleadow, R.M. and B.L. Møller. 2014. Cyanogenic glycosides: Synthesis, physiology, and phenotypic plasticity. Annu. Rev. Plant Biol. 65:155-185.

Godini, A., M. Palasciano, G. Ferrara, S. Camposeo, and A. Pacifico. 2008. On the advancement of bud break and fruit ripening induced by hydrogen cyanamide (Dormex $\left.{ }^{\circledR}\right)$ in sweet cherry: A three-year study. Acta Hort. 795:469-478.

Gookin, T.E., D.A. Hunter, and M.S. Reid. 2003. Temporal analysis of alpha and beta-expansin expression during floral opening and senescence. Plant Sci. 164:769-781.

Halaly, T., X. Pang, T. Batikoff, O. Crane, A. Keren, J. Venkateswari, A. Ogrodovitch, A. Sadka, S. Lavee, and E. Or. 2008. Similar mechanisms might be triggered by alternative external stimuli that induce dormancy release in grape buds. Planta 228:79-88.

Hellemans, J., G. Mortier, A. De Paepe, F. Speleman, and J. Vandesompele. 2007. qBase relative quantification framework and software for management and automated analysis of real-time quantitative PCR data. Genome Biol. 8:R19.

Henzell, R.F., M.R. Briscoe, and I. Gravett. 1992. Improving kiwifruit vine productivity with plant growth regulators. Acta Hort. 297:345-350.

Huang, D.W., B.T. Sherman, and R.A. Lempicki. 2009. Systematic and integrative analysis of large gene lists using DAVID bioinformatics resources. Nat. Protoc. 4:44-57.

Ionescu, I.A., B.L. Møller, and R. Sánchez-Pérez. 2017a. Chemical control of flowering time. J. Expt. Bot. 68:369-382.

Ionescu, I.A., G. López-Ortega, M. Burow, A. Bayo-Canha, A. Junge, O. Gericke, B.L. Møller, and R. Sánchez-Pérez. 2017b. Transcriptome and metabolite changes during hydrogen cyanamide-induced floral bud break in sweet cherry. Front. Plant Sci. 8:1233.

Jackson, J.E. and M. Bepete. 1995. The effect of hydrogen cyanamide (Dormex) on flowering and cropping of different apple cultivars under tropical conditions of sub-optimal winter chilling. Scientia Hort. 60:293-304.

Jiménez, S., G.L. Reighard, and D.G. Bielenberg. 2010. Gene expression of DAM5 and DAM6 is suppressed by chilling temperatures and inversely correlated with bud break rate. Plant Mol. Biol. 73:157-167.

Joo, J.H., Y.S. Bae, and J.S. Lee. 2001. Role of auxin-induced reactive oxygen species in root gravitropism. Plant Physiol. 126:1055-1060. Kanehisa, M., M. Furumichi, M. Tanabe, Y. Sato, and K. Morishima. 2017. KEGG: New perspectives on genomes, pathways, diseases and drugs. Nucleic Acids Res. 45:D353-D361.

Keilin, T., X. Pang, J. Venkateswari, T. Halaly, O. Crane, A. Keren, A. Ogrodovitch, R. Ophir, H. Volpin, D. Galbraith, and E. Or. 2007. Digital expression profiling of a grape-bud EST collection leads to new insight into molecular events during grape-bud dormancy release. Plant Sci. 173:446-457.

Kobayashi, M., I. Ohura, K. Kawakita, N. Yokota, M. Fujiwara, K. Shimamoto, N. Doke, and H. Yoshioka. 2007. Calcium-dependent protein kinases regulate the production of reactive oxygen species by potato NADPH Oxidase. Plant Cell 19:1065-1080.

Kuroda, H., T. Sugiura, and D. Ito. 2002. Changes in hydrogen peroxide content in flower buds of japanese pear (Pyrus pyrifolia Nakai) in relation to breaking of endodormancy. J. Jpn. Soc. Hort. Sci. 71:610-616.

Lang, G.A., J.D. Early, G.C. Martin, and R.L. Darnell. 1987. Endo-, para-, and ecodormancy: Physiological terminology and classification for dormancy research. HortScience 22:371-377. 
Li, R., P.C. Bonham-Smith, and J. King. 2001. Molecular characterization and regulation of formate dehydrogenase in Arabidopsis thaliana. Can. J. Bot. 79:796-804.

Li, B. and C.N. Dewey. 2011. RSEM: Accurate transcript quantification from RNA-Seq data with or without a reference genome. BMC Bioinformatics 12:323.

Liszkay, A., E. van der Zalm, and P. Schopfer. 2004. Production of reactive oxygen intermediates $\left(\mathrm{O}_{2}{ }^{--}, \mathrm{H}_{2} \mathrm{O}_{2}\right.$, and $\left.{ }^{\circ} \mathrm{OH}\right)$ by maize roots and their role in wall loosening and elongation growth. Plant Physiol. 136:3114-3123.

May, M.J., T. Vernoux, C. Leaver, N.V. Montagu, and D. Inzé. 1998. Glutathione homeostasis in plants: Implications for environmental sensing and plant development. J. Expt. Bot. 49:649-667.

Mittler, R. 2002. Oxidative stress, antioxidants and stress tolerance. Trends Plant Sci. 7:405-410.

Mittler, R., S. Vanderauwera, M. Gollery, and F. Van Breusegem. 2004. Reactive oxygen gene network of plants. Trends Plant Sci. 9:490-498.

Mittler, R., S. Vanderauwera, N. Suzuki, G. Miller, V.B. Tognetti, K. Vandepoele, M. Gollery, V. Shulaev, and F. Van Breusegem. 2011. ROS signaling: The new wave? Trends Plant Sci. 16:300309.

Mittler, R. 2017. ROS are good. Trends Plant Sci. 22:11-19.

Morant, A.V., K. Jørgensen, C. Jørgensen, S.M. Paquette, R. SánchezPérez, B.L. Møller, and S. Bak. 2008. $\beta$-Glucosidases as detonators of plant chemical defense. Phytochemistry 69:1795-1813.

Morgan, K. and M. Olmstead. 2013. Peach orchard establishment and production planning budgets for Florida. Proc. Florida State Hort. Soc. 126:35-42.

Müller, K., A. Linkies, R.A.M. Vreeburg, S.C. Fry, A. KriegerLiszkay, and G. Leubner-Metzger. 2009. In vivo cell wall loosening by hydroxyl radicals during cress seed germination and elongation growth. Plant Physiol. 150:1855-1865.

National Center for Biotechnology Information. 2018. A tool for finding specific primers. 1 Dec. 2018. <http://www.ncbi.nlm.nih. gov/tools/primer-blast/index.cgi?LINK_LOC=BlastHome $>$.

Nir, G., Y. Shulman, L. Fanberstein, and S. Lavee. 1986. Changes in the activity of catalase (EC 1.11.1.6) in relation to the dormancy of grapevine (Vitis vinifera L.) buds. Plant Physiol. 81:1140-1142.

Ogasawara, Y., H. Kaya, G. Hiraoka, F. Yumoto, S. Kimura, Y. Kadota, H. Hishinuma, E. Senzaki, S. Yamagoe, K. Nagata, M. Nara, K. Suzuki, M. Tanokura, and K. Kuchitsu. 2008. Synergistic activation of the Arabidopsis NADPH oxidase AtrbohD by $\mathrm{Ca}^{2+}$ and phosphorylation. J. Biol. Chem. 283:8885-8892.

Okie, W.R. 1998. Handbook of peach and nectarine varieties: Performance in the southeastern United States and index of names. U.S. Dept. Agr., Washington, DC.

Olmstead, M., J. Chaparro, P. Andersen, J. Williamson, and J. Ferguson. 2016. Florida peach and nectarine varieties. Univ. Florida/Inst. Food Agr. Sci. Ext. Digital Info. Source MG374.

Olmstead, M. 2014. Application of hydrogen cyanamide to increase bloom uniformity in low-chill peaches: A preliminary report. Proc. Florida State Hort. Soc. 127:18-20.

Or, E., I. Vilozny, Y. Eyal, and A. Ogrodovitch. 2000. The transduction of the signal for grape bud dormancy breaking induced by hydrogen cyanamide may involve the SNF-like protein kinase GDBRPK. Plant Mol. Biol. 43:483-494.

Or, E., I. Vilozny, A. Fennell, Y. Eyal, and A. Ogrodovitch. 2002. Dormancy in grape buds: Isolation and characterization of catalase cDNA and analysis of its expression following chemical induction of bud dormancy release. Plant Sci. 162:121-130.

Pandolfi, P.P., F. Sonati, R. Rivi, P. Mason, F. Grosveld, and L. Luzzatto. 1995. Targeted disruption of the housekeeping gene encoding glucose 6-phosphate dehydrogenase (G6PD): G6PD is dispensable for pentose synthesis but essential for defense against oxidative stress. EMBO J. 14:5209-5215.

Perata, P. and A. Alpi. 1993. Plant responses to anaerobiosis. Plant Sci. 93:1-17.
Pérez, F.J., R. Vergara, and E. Or. 2009. On the mechanism of dormancy release in grapevine buds: A comparative study between hydrogen cyanamide and sodium azide. Plant Growth Regulat. 59:145-152.

Pfaffl, M.W. 2001. A new mathematical model for relative quantification in real-time RT-PCR. Nucleic Acids Res. 29:e45.

Piotrowski, M., S. Schönfelder, and E.W. Weiler. 2001. The Arabidopsis thaliana isogene NIT4 and its orthologs in tobacco encode $\beta$-cyano-L-alanine hydratase/nitrilase. J. Biol. Chem. 276:26162621.

Reczek, C.R. and N.S. Chandel. 2015. ROS-dependent signal transduction. Curr. Opin. Cell Biol. 33:8-13.

Rio, D.C. 2015. Denaturation and electrophoresis of RNA with formaldehyde. Cold Spring Harb. Protoc. 2015:306-313.

Rodriguez-A, J., W.B. Sherman, R. Scorza, M. Wisniewski, and W.R. Okie. 1994. 'Evergreen' peach, its inheritance and dormant behavior. J. Amer. Soc. Hort. Sci. 119:789-792.

Rohde, A. and R.P. Bhalerao. 2007. Plant dormancy in the perennial context. Trends Plant Sci. 12:217-223.

Rouhier, N. 2010. Plant glutaredoxins: Pivotal players in redox biology and iron-sulphur centre assembly. New Phytol. 186:365-372.

Salvemini, F., A. Franzé, A. Iervolino, S. Filosa, S. Salzano, and M.V. Ursini. 1999. Enhanced glutathione levels and oxidoresistance mediated by increased glucose-6-phosphate dehydrogenase expression. J. Biol. Chem. 274:2750-2757.

Scalabrelli, G., R. Viti, and F. Cinelli. 1991. Change in catalase activity and dormancy of apricot buds in response to chilling. Acta Hort. 293:267-274.

Scarpeci, T.E., M.I. Zanor, N. Carrillo, B. Mueller-Roeber, and E.M. Valle. 2008. Generation of superoxide anion in chloroplasts of Arabidopsis thaliana during active photosynthesis: A focus on rapidly induced genes. Plant Mol. Biol. 66:361-378.

Shirota, F.N., E.G. Demaster, and H.T. Nagasawa. 1987. Cyanide is a product of the catalase-mediated oxidation of the alcohol deterrent agent, cyanamide. Toxicol. Lett. 37:7-12.

Shulman, Y., G. Nir, and S. Lavee. 1986. Oxidative processes in bud dormancy and the use of hydrogen cyanamide in breaking dormancy. Acta Hort. 179:141-148.

Siegień, I. and R. Bogatek. 2006. Cyanide action in plants - From toxic to regulatory. Acta Physiol. Plant. 28:483-497.

Singerman, A., M. Burani-Arouca, and M. Olmstead. 2017. Establishment and production costs for peach orchards in Florida: Enterprise budget and profitability analysis. Univ. Florida/Inst. Food Agr. Sci. Ext. Digital Info. Source FE1016.

Sudawan, B., C.-S. Chang, H. Chao, M.S.B. Ku, and Y. Yen. 2016. Hydrogen cyanamide breaks grapevine bud dormancy in the summer through transient activation of gene expression and accumulation of reactive oxygen and nitrogen species. BMC Plant Biol. 16:202.

Supek, F., M. Bošnjak, N. Škunca, and T. Šmuc. 2011. REVIGO summarizes and visualizes long lists of gene ontology terms. PLoS One 6:e21800.

Tani, E., A.N. Polidoros, E. Flemetakis, C. Stedel, C. Kalloniati, K. Demetriou, P. Katinakis, and A.S. Tsaftaris. 2009. Characterization and expression analysis of AGAMOUS-like SEEDSTICK-like and SEPALLATA-like MADS-box genes in peach (Prunus persica) fruit. Plant Physiol. Biochem. 47:690-700.

Thimm, O., O. Bläsing, Y. Gibon, A. Nagel, S. Meyer, P. Krüger, J. Selbig, L.A. Müller, S.Y. Rhee, and M. Stitt. 2004. MAPMAN: A user-driven tool to display genomics data sets onto diagrams of metabolic pathways and other biological processes. Plant J. 37:914939.

Tian, T., Y. Liu, H. Yan, Q. You, X. Yi, Z. Du, W. Xu, and Z. Su. 2017. AgriGO v2.0: A GO analysis toolkit for the agricultural community 2017 update. Nucleic Acids Res. 45:W122-W129.

Tong, Z., Z. Gao, F. Wang, J. Zhou, and Z. Zhang. 2009. Selection of reliable reference genes for gene expression studies in peach using real-time PCR. BMC Mol. Biol. 10:71. 
Turrens, J.F. 2003. Mitochondrial formation of reactive oxygen species. J. Physiol. 552:335-344.

Usadel, B., A. Nagel, O. Thimm, H. Redestig, O.E. Blaesing, N. Palacios-Rojas, J. Selbig, J. Hannemann, M.C. Piques, D. Steinhauser, W.-R. Scheible, Y. Gibon, R. Morcuende, D. Weicht, S. Meyer, and M. Stitt. 2005. Extension of the visualization tool MapMan to allow statistical analysis of arrays, display of corresponding genes, and comparison with known responses. Plant Physiol. 138:1195-1204.

Vanlerberghe, G.C. and L. McIntosh. 1997. Alternative oxidase: From gene to function. Annu. Rev. Plant Biol. 48:703-734.

Verde, I., J. Jenkins, L. Dondini, S. Micali, G. Pagliarani, E. Vendramin, R. Paris, V. Aramini, L. Gazza, L. Rossini, D. Bassi, M. Troggio, S. Shu, J. Grimwood, S. Tartarini, M.T. Dettori, and J. Schmutz. 2017. The peach v2.0 release: High-resolution linkage mapping and deep resequencing improve chromosome-scale assembly and contiguity. BMC Genomics 18:225.

Viti, R., S. Bartolini, and G.C. Zanol. 2012. Biological changes and active oxygen-scavenging enzymes activities in apricot (Prunus armeniaca L.) flower buds during dormancy transitions. Acta Hort. 940:331-339.
Weinberger, J.H. 1950. Chilling requirements of peach varieties. Proc. Amer. Soc. Hort. Sci. 56:122-128.

Williamson, J.F., G. Krewer, B.E. Maust, and E.P. Miller. 2002. Hydrogen cyanamide accelerates vegetative budbreak and shortens fruit development period of blueberry. HortScience 37:539-542.

Yamada, K., R. Takahashi, C. Fujitani, K. Mishima, M. Yoshida, D.C. Joyce, and S. Yamaki. 2009. Cell wall extensibility and effect of cellwall-loosening proteins during rose flower opening. J. Jpn. Soc. Hort. Sci. 78:242-251.

Yamane, H., T. Ooka, H. Jotatsu, Y. Hosaka, R. Sasaki, and R. Tao. 2011. Expressional regulation of PpDAM5 and PpDAM6, peach (Prunus persica) dormancy-associated MADS-box genes, by low temperature and dormancy-breaking reagent treatment. J. Expt. Bot. 62:3481-3488.

Zhang, L., Y. Xu, and R. Ma. 2008. Molecular cloning, identification, and chromosomal localization of two MADS box genes in peach (Prunus persica). J. Genet. Genomics 35:365-372.

Zhuang, W., Z. Gao, L. Wang, W. Zhong, Z. Ni, and Z. Zhang. 2013. Comparative proteomic and transcriptomic approaches to address the active role of $\mathrm{GA}_{4}$ in japanese apricot flower bud dormancy release. J. Expt. Bot. 64:4953-4966. 
A

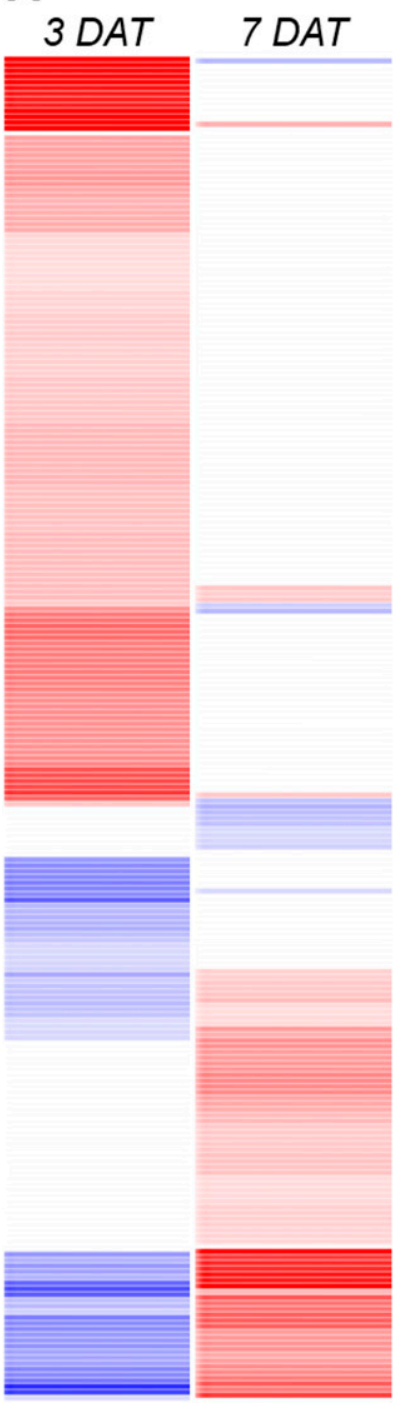

B

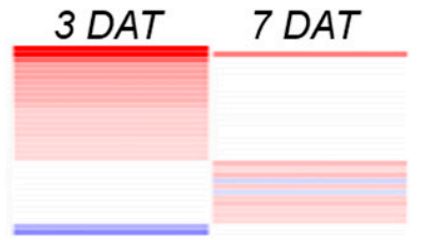

C
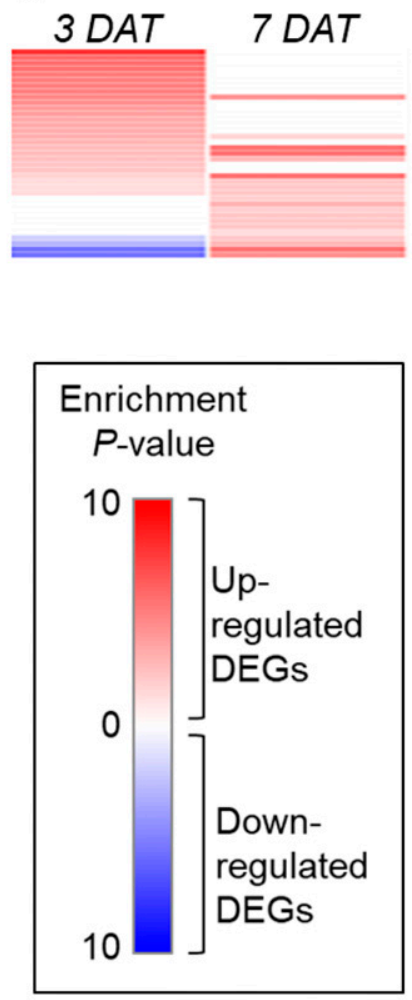

Supplemental Fig. 1. Significant gene ontology (GO) terms of upregulated and downregulated differentially expressed genes (DEGs), indicated by red and blue, respectively, in buds of 'TropicBeauty' peach trees treated with $1 \%(\mathrm{v} / \mathrm{v})$ hydrogen cyanamide compared with nontreated control trees at 3 and $7 \mathrm{~d}$ after treatment (DAT) for the biological process (A), molecular function (B), and cellular component (C). The intensity of the color indicates the enrichment values [absolute values of the logarithm (to the base of 10) of false discovery rate-corrected $P$ values based on Fisher's exact test for GO enrichment analysis] of individual GO terms. 

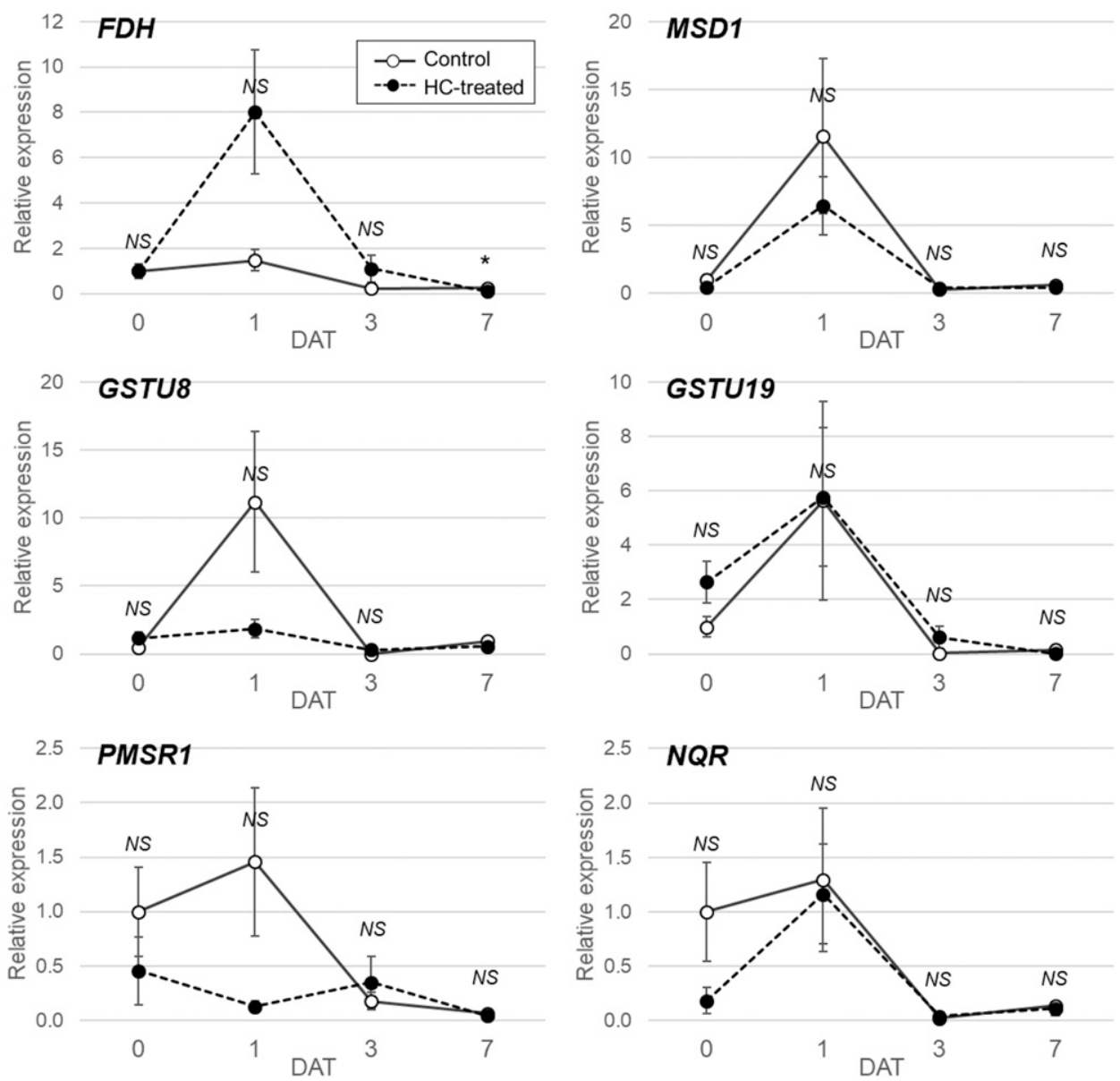

Supplemental Fig. 2. Relative expressions of formate dehydrogenase (FDH), manganese superoxide dismutase1 (MSD1), glutathione s-transferase tau8 (GSTU8), glutathione s-transferase tau19 (GSTU19), peptide methionine sulfoxide reductase1 (PMSR1), and nadph:quinone oxidoreductase (NQR) in buds of nontreated control 'TropicBeauty' peach trees (solid lines with white circles) and trees treated with $1 \%$ (v/v) hydrogen cyanamide [HC (broken lines with black circles)] at 0 , 1,3 , and $7 \mathrm{~d}$ after treatment (DAT) analyzed using real-time quantitative polymerase chain reaction (qPCR); data are means $\pm \mathrm{SE}$ of four biological replicates; asterisks indicate significant differences based on Student's $t$ test between control and HC-treated trees on the same day; NS = no significant difference. 

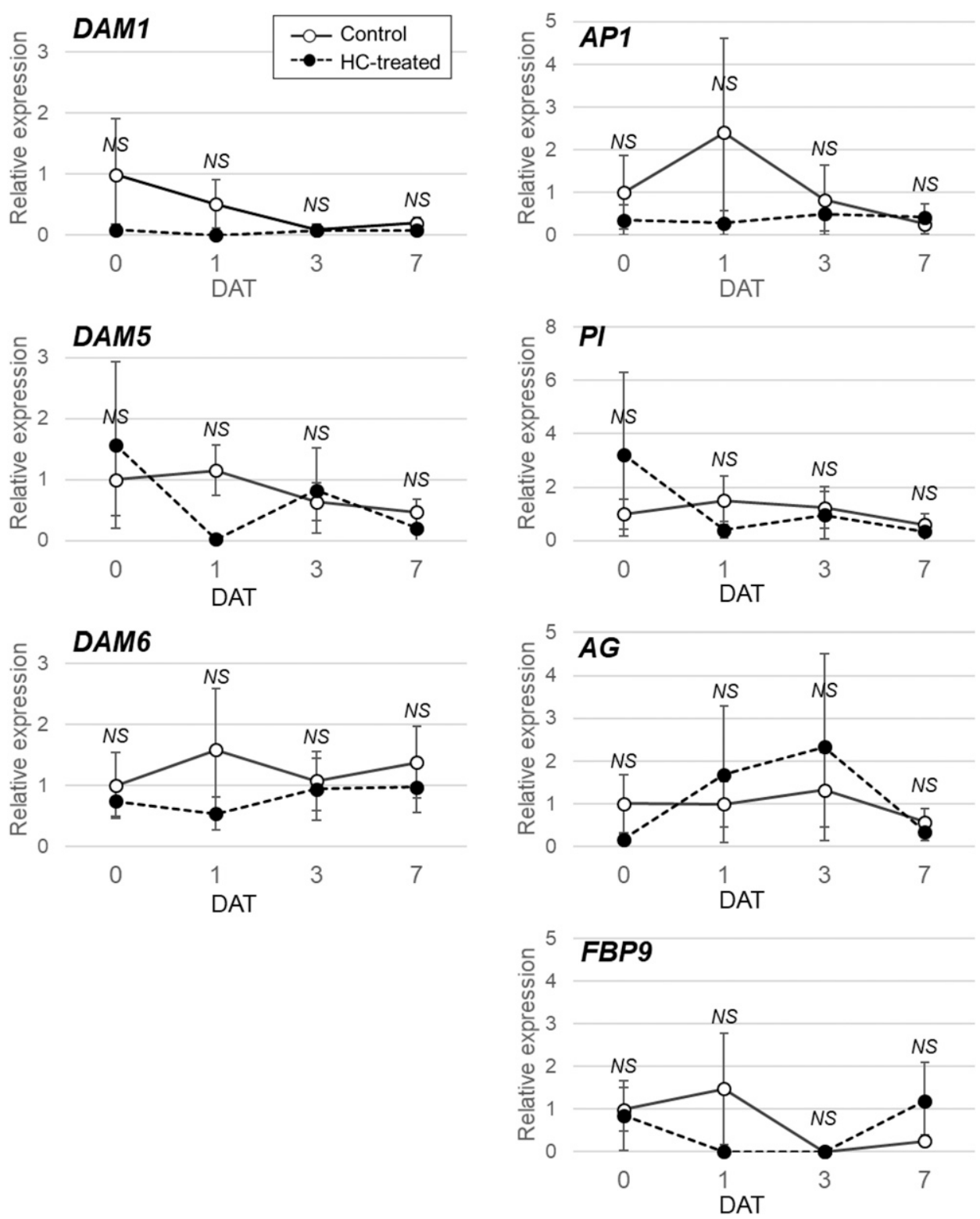

Supplemental Fig. 3. Relative expression of dormancy-associated MADS-box 1 (DAM1), DAM5, and DAM6, apetala1 (AP1), pistillata (PI), agamous (AG), and floral binding protein9 (FBP9) in buds of nontreated control 'TropicBeauty' peach trees (solid lines with white circles) and trees treated with $1 \%$ (v/v) hydrogen cyanamide [HC (broken lines with black circles)] at $0,1,3$, and $7 \mathrm{~d}$ after treatment (DAT) analyzed using real-time quantitative polymerase chain reaction (qPCR); data are means \pm SE of four biological replicates; NS = no significant difference based on Student's $t$ test between control and HC-treated trees on the same day. 
Supplemental Table 1. Summary of RNA sequencing read information for 'TropicBeauty' peach bud samples of nontreated control trees and trees treated with $1 \%(\mathrm{v} / \mathrm{v})$ hydrogen cyanamide $(\mathrm{HC})$ at 3 and $7 \mathrm{~d}$ after treatment (DAT).

\begin{tabular}{lcccrr}
\hline Treatment & DAT & Biological replicate & Raw reads & Trimmed reads & Transcriptome alignments \\
\hline Control & 3 & 1 & 36276808 & 29884999 & 12009560 \\
Control & 3 & 2 & 37238081 & 30307565 & 4741934 \\
Control & 3 & 3 & 39282984 & 33706513 & 37164310 \\
Control & 3 & 4 & 38817678 & 33371934 & 45529258 \\
HC-treated & 3 & 1 & 45601653 & 40573824 & 51555728 \\
HC-treated & 3 & 2 & 37073677 & 32270752 & 21196510 \\
HC-treated & 3 & 3 & 45042543 & 39242321 & 73268566 \\
HC-treated & 3 & 4 & 33841769 & 29584090 & 19589144 \\
Control & 7 & 1 & 46945094 & 38456093 & 1883158 \\
Control & 7 & 2 & 48016978 & 39702896 & 12044624 \\
Control & 7 & 3 & 35387093 & 27174001 & 7074268 \\
Control & 7 & 4 & 36725383 & 31801548 & 31182584 \\
HC-treated & 7 & 1 & 39365521 & 34862495 & 57544194 \\
HC-treated & 7 & 2 & 45332061 & 39025325 & 12125968 \\
HC-treated & 7 & 3 & 43556959 & 36183179 & 8804520 \\
HC-treated & 7 & 4 & 38107726 & 33323015 & 11618240 \\
\hline
\end{tabular}


Supplemental Table 2. Gene-specific primer sequences for relative gene expression analysis of 'TropicBeauty' peach with quantitative real-time polymerase chain reaction.

\begin{tabular}{|c|c|c|c|c|}
\hline Gene & Description & Accession no. & $\begin{array}{c}\text { Forward and reverse primer* } \\
\text { sequences }\left(5^{\prime} \text { to } 3^{\prime}\right)\end{array}$ & $\begin{array}{l}\text { Amplicon } \\
\text { size (bp) }\end{array}$ \\
\hline \multirow[t]{2}{*}{$\overline{F D H}$} & Formate dehydrogenase & XM_020559076.1 & AATGCTCGAGGGGCAATCAT & 106 \\
\hline & & & TCGGAGCTGGTTGTGGATTC & \\
\hline \multirow[t]{2}{*}{ MSD1 } & Manganese superoxide dismutase & XM_007218235.2 & AGATCAATGCAGAAGGCGCT & 107 \\
\hline & & & TGGGTCCTGATTTGCAGTGG & \\
\hline \multirow[t]{2}{*}{ GSTU8 } & Glutathione S-transferase TAU8 & XM_007208120.2 & ATGAGACTTGGAAGCAGGGTTT & 138 \\
\hline & & & CATGTTCTTCACTCCCCCAGC & \\
\hline \multirow[t]{2}{*}{ GSTU19 } & Glutathione S-transferase TAU19 & XM_020561747.1 & AGTCCCTCATTGCGGTTCAG & 151 \\
\hline & & & GTTGTCCACACCTTCCTCCC & \\
\hline \multirow[t]{2}{*}{ PMSR1 } & Peptide methionine sulfoxide reductase & XM_007209566.2 & TCTCTGTCTTTTGGGCTCGT & 151 \\
\hline & & & TTCAACTCCACTTGCTTTGCC & \\
\hline \multirow[t]{2}{*}{$N Q R$} & NADPH:quinone oxidoreductase & XM_007218384.2 & CGGTCGTTGAAGCATTTCGT & 149 \\
\hline & & & GGCAGCCTTATCACCCCAAA & \\
\hline \multirow[t]{2}{*}{$D A M 1$} & Dormancy-associated MADS-box gene & XM_020563603.1 & GCGCACATAAATGGTGCTGA & 105 \\
\hline & & & GCGGCTCTTCTCCTCAAGTT & \\
\hline \multirow[t]{2}{*}{ DAM5 } & Dormancy-associated MADS-box gene & XM_007223804.2 & TGTGTGAATCTGAGGTGGCA & 118 \\
\hline & & & TCGACACCATTTATGTCTGCG & \\
\hline \multirow[t]{2}{*}{ DAM6 } & Dormancy-associated MADS-box gene & XM_007227500.2 & GATGACTGCTCCGATGTCACT & 139 \\
\hline & & & CACTACTGATGCGTCTCACA & \\
\hline \multirow[t]{2}{*}{$A P 1$} & Class A floral organ identity gene APETALA1 & XM_007223759.2 & AGAAGATCAAGGAGAAGGAGAAG & 102 \\
\hline & & & TTAGACATGGAAGCGGCTGT & \\
\hline \multirow[t]{2}{*}{$P I$} & Class B floral organ identity gene PISTILLATA & XM_020554792.1 & GAGCACTGGAAGAGGAGCAT & 151 \\
\hline & & & GGCTGAACACGAAAGGCAAA & \\
\hline \multirow[t]{2}{*}{$A G$} & Class $\mathrm{C}$ floral organ identity gene $A G A M O U S$ & XM_007211863.2 & TTCACGCCAAGATCCGATGG & 130 \\
\hline & & & CTTGACAAAGTGCCCGTAGC & \\
\hline \multirow[t]{2}{*}{ FBP9 } & Class E floral organ identity gene FLORAL & XM_007209443.2 & TCCAGCCGTGGCAAACTTTA & 107 \\
\hline & BINDING PROTEIN9 & & ACTGGTCTGTTGGCTTCCAG & \\
\hline \multirow[t]{2}{*}{$T E F 2$} & Translation elongation factor 2 & XM_007214747.2 & TGTAGTCAGCATCCTGTGCC & 108 \\
\hline & & & ACCAGCAATGGCAGAAATGG & \\
\hline \multirow[t]{2}{*}{$U B Q$} & Ubiquitin & XM_020559001.1 & ACTACTGTGGTAAGTGCGGC & 119 \\
\hline & & & AACCAACTGTTCAGCACCCT & \\
\hline
\end{tabular}


Supplemental Table 3. Numbers of differentially expressed genes (DEGs) encoding the members of transcription factor (TF) families in buds of 'TropicBeauty' peach trees treated with $1 \%(\mathrm{v} / \mathrm{v})$ hydrogen cyanamide compared with nontreated control trees at 3 and $7 \mathrm{~d}$ after treatment (DAT).

\begin{tabular}{|c|c|c|c|c|}
\hline TF family & $\begin{array}{c}\text { Upregulated } \\
\text { DEGs at } 3 \text { DAT (no.) }\end{array}$ & $\begin{array}{c}\text { Downregulated } \\
\text { DEGs at } 3 \text { DAT (no.) }\end{array}$ & $\begin{array}{c}\text { Upregulated } \\
\text { DEGs at } 7 \text { DAT (no.) }\end{array}$ & $\begin{array}{c}\text { Downregulated } \\
\text { DEGs at } 7 \text { DAT (no.) }\end{array}$ \\
\hline AP2 & 0 & 0 & 1 & 0 \\
\hline $\mathrm{ARF}$ & 0 & 0 & 2 & 1 \\
\hline bHLH & 1 & 8 & 2 & 3 \\
\hline $\mathrm{C} 2 \mathrm{H} 2$ & 0 & 4 & 0 & 0 \\
\hline ERF & 1 & 9 & 3 & 3 \\
\hline GATA & 0 & 1 & 0 & 1 \\
\hline GRF & 0 & 2 & 0 & 0 \\
\hline HSF & 1 & 1 & 0 & 1 \\
\hline LBD & 1 & 3 & 0 & 0 \\
\hline MIKC_MADS & 0 & 3 & 1 & 2 \\
\hline MYB & 4 & 6 & 3 & 2 \\
\hline MYB-related & 1 & 3 & 0 & 5 \\
\hline NAC & 3 & 1 & 2 & 3 \\
\hline WRKY & 4 & 1 & 2 & 1 \\
\hline YABBY & 0 & 1 & 2 & 0 \\
\hline
\end{tabular}

